\title{
Types of topological surface states in nodal noncentrosymmetric superconductors
}

\author{
Andreas P. Schnyder, 1 , f P. M. R. Brydon, 2 , and Carsten Timm², 用 \\ ${ }^{1}$ Max-Planck-Institut für Festkörperforschung, Heisenbergstrasse 1, D-70569 Stuttgart, Germany \\ ${ }^{2}$ Institut für Theoretische Physik, Technische Universität Dresden, D-01062 Dresden, Germany
}

(Dated: November 2, 2018)

\begin{abstract}
Nodal noncentrosymmetric superconductors have topologically nontrivial properties manifested by protected zero-energy surface states. Specifically, it was recently found that zero-energy surface flat bands of topological origin appear at their surface. We show that the presence of certain inversion-type lattice symmetries can give rise to additional topological features of the gap nodes, resulting in surface states forming one-dimensional arcs connecting the projections of two nodal rings. In addition, we demonstrate that Majorana surface states can appear at time-reversal-invariant momenta of the surface Brillouin zone, even when the system is not fully gapped in the bulk. Within a continuum theory we derive the topological invariants that protect these different types of zero-energy surface states. We independently derive general conditions for the existence of zero-energy surface bound states using the complementary quasiclassical scattering theory, explicitly taking into account the effects of spin-orbit splitting of the bands. We compute surface bound-state spectra for various crystal point-group symmetries and orbital-angular-momentum pairing states. Finally, we examine the signatures of the arc surface states and of the zero-energy surface flat bands in tunneling-conductance spectra and dicuss how topological phase transitions in noncentrosymmetric superconductors could be observed in experiments.
\end{abstract}

PACS numbers: 74.50.+r, 74.20.Rp, 74.25.F-, 03.65.vf

\section{INTRODUCTION}

Systems with strong spin-orbit coupling (SOC) have recently attracted a great deal of attention. Prime examples are topological insulators, where strong spin-orbit interactions give rise to a non-trivial band topology, leading to topologically protected zero-energy surface states. $1 \cdot\left[\frac{1}{3}\right.$ Another class of compounds for which SOC plays an important role are superconductors without inversion symmetry ${ }^{4 / 5}$ In these remarkable materials, Rashba-type antisymmetric spin-orbit interactions lift the spin degeneracy of the electronic bands and generate complex spin textures in the electron Bloch functions. In the superconducting state, the antisymmetric SOC gives rise to the admixture of even-parity spin-singlet and odd-parity spin-triplet pairing components and, importantly, allows a non-trivial topology of the Bogoliubov-quasiparticle wavefunctions. ${ }^{69}$ Akin to topological insulators, this nontrivial wavefunction topology results in various types of protected zero-energy states at the edge or surface of noncentrosymmetric superconductors (NCSs). ${ }^{[8] 14}$ For instance, a fully gapped NCS with nontrivial topology supports linearly dispersing helical Majorana modes at its boundary ${ }^{89115+20} \mathrm{In}$ three-dimensional systems, the stability of these Majorana surface states is protected by an integer $(\mathbb{Z})$ topological invariant, i.e., the three-dimensional winding number,, 9151 whereas in two-dimensional systems a binary $\left(\mathbb{Z}_{2}\right)$ topological number guarantees the robustness of the edge modes. ${ }^{[117 / 21} \mid 23$

Remarkably, topologically protected zero-energy boundary modes also occur in NCSs with line nodes ${ }^{9-14}$ In particular, it has recently been shown that dispersionless zero-energy states (i.e., flat bands) of topological origin generically appear at the surface of three-dimensional nodal NCSs. ${ }^{910}$ These zero-energy flat bands are confined to regions of the twodimensional surface Brillouin zone (BZ) that are bounded by the projections of the nodal lines of the bulk gap ${ }^{[24}$ The topological protection of these dispersionless boundary states is linked to the topological characteristics of the nodal gap structure via a bulk-boundary correspondence. In fact, the stability of both the dispersionless zero-energy surface states and the line nodes of the bulk gap is ensured by the conservation of the same integer topological invariant, namely the one-dimensional winding number. Apart from these twodimensional surface flat bands, certain NCSs also support zero-energy boundary states that form one-dimensional open arcs in the surface BZ, connecting the projection of two nodal rings! ${ }^{10|20| 25 \mid 29]}$ Moreover, it has recently been reported that Majorana surface states can occur at time-reversal-invariant momenta of the surface BZ, 9114 even if the superconductor is not fully gapped in the bulk.

Topological surface states are generic features of NCSs whose spin-triplet pairing component is at least as strong as the spin-singlet one. It is therefore quite reasonable to expect that these zero-energy boundary states occur in the superconducting state of the heavy fermion compounds $\mathrm{CePt}_{3} \mathrm{Si}_{2}{ }^{30} \mathrm{CeRhSi}_{3},{ }_{11}$ and $\mathrm{CeIrSi}_{3},{ }_{32}$ as well as in $\mathrm{Y}_{2} \mathrm{C}_{3}, \frac{33}{33}$

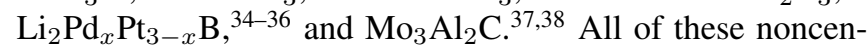
trosymmetric materials show strong spin-orbit interactions, with the spin-orbit band splitting far exceeding the superconducting energy scale .5 For the study of surface phenomena in NCSs it is thus important to explicitly account for the strong SOC, previous works! $1020 \mid 25+28$

The main aim of this paper is to provide a comprehensive classification of the topological features of three-dimensional nodal NCSs. In particular, we demonstrate that the presence of certain inversion-type symmetries gives rise to additional topological features of the gap line nodes. These features are characterized by a two-dimensional $\mathbb{Z}_{2}$ topological invariant and manifest themselves on the surface as one-dimensional arcs of zero-energy states, terminating at the projection of the nodal lines onto the surface BZ. We also examine the appearance of Majorana surface states at time-reversal-invariant mo- 
menta of the surface BZ and show that the topological properties of these linearly dispersing modes are described by a one-dimensional $\mathbb{Z}_{2}$ invariant. Using a continuum model of NCSs, we derive expressions for the $\mathbb{Z}_{2}$ invariants and the winding number that protect the Majorana modes, the arc surface states, and the surface flat bands, respectively.

We illustrate these topological features by investigating the surface-bound-state spectrum and the tunneling conductance of NCSs using quasiclassical scattering theory. Allowing for non-negligible SOC, we consider three different experimentally relevant crystal point-group symmetries and explore the effects of pairing with higher orbital angular momenta [e.g., $\left(d_{x^{2}-y^{2}}+f\right)$-wave pairing]. We find that higher-orbital-angular-momentum pairing leads to additional topologically stable line nodes in the bulk gap. Correspondingly, there appear extra surface flat bands associated with these additional nodal lines. We show that the surface flat bands and the arc states leave unique signatures in the tunneling-conductance spectra. Finally, we investigate topological phase transitions in NCSs, i.e., zero-temperature quantum phase transitions where the momentum-space topology of the quasiparticle spectrum changes abruptly as a function of the singlet-to-triplet ratio in the pairing amplitude. We argue that anomalies in the density of states and the tunneling conductance provide experimental fingerprints of these zero-temperature phase transitions.

The remainder of this paper is organized as follows. Section $\Pi$ discusses the model Hamiltonian and its symmetries. In Sec. III we derive the topological invariants characterizing topological properties of both the nodal lines and the surface states and give a detailed discussion of the topological criteria for the existence of zero-energy surface states. By use of quasiclassical scattering theory, we derive in Sec. IV] general conditions for the occurrence of surface bound states in terms of sign changes of the superconducting gap functions across the Fermi surface. We show that these conditions are in perfect agreement with the topological criteria given in Sec. III. In addition, we present in Sec. IV surface-bound state spectra for three different crystal point-group symmetries and for various surface orientations. In Sec. V A we compute the tunneling conductance between a normal metal and an NCS, identify the signatures of the zero-energy surface flat bands and the arc surface states in the tunneling spectra, and discuss topological phase transitions. Our conclusions and outlook are given in Sec.VI.

\section{MODEL HAMILTONIAN AND SYMMETRIES}

We consider a three-dimensional single-band BCS superconductor with noncentrosymmetric crystal structure and Rashba-type SOC. On a phenomenological level, such a superconductor is described by the Bogoliubov-de Gennes Hamiltonian $\mathcal{H}=\frac{1}{2} \sum_{\mathbf{k}} \Psi_{\mathbf{k}}^{\dagger} \mathcal{H}(\mathbf{k}) \Psi_{\mathbf{k}}$, with

$$
\mathcal{H}(\mathbf{k})=\left(\begin{array}{cc}
h(\mathbf{k}) & \Delta(\mathbf{k}) \\
\Delta^{\dagger}(\mathbf{k}) & -h^{\mathrm{T}}(-\mathbf{k})
\end{array}\right)
$$

and $\Psi_{\mathbf{k}}=\left(c_{\mathbf{k} \uparrow}, c_{\mathbf{k} \downarrow}, c_{-\mathbf{k} \uparrow}^{\dagger}, c_{-\mathbf{k} \downarrow}^{\dagger}\right)^{\mathrm{T}}$, where $c_{\mathbf{k} \sigma}^{\dagger}\left(c_{\mathbf{k} \sigma}\right)$ denotes the electron creation (annihilation) operator with momentum $\mathbf{k}$ and spin $\sigma$. The normal-state dispersion of the electrons in the spin basis is given by

$$
h(\mathbf{k})=\varepsilon_{\mathbf{k}} \sigma_{0}+\mathbf{g}_{\mathbf{k}} \cdot \boldsymbol{\sigma},
$$

where $\varepsilon_{\mathbf{k}}=\hbar^{2} \mathbf{k}^{2} /(2 m)-\mu, \mathbf{g}_{\mathbf{k}}$ denotes the SOC potential, $\boldsymbol{\sigma}=\left(\sigma_{x}, \sigma_{y}, \sigma_{z}\right)^{\mathrm{T}}$ are the three Pauli matrices, and $\sigma_{0}$ stands for the $2 \times 2$ unit matrix. In the so-called helicity basis the normal-state Hamiltonian (1b) takes diagonal form, $\tilde{h}(\mathbf{k})=$ $\operatorname{diag}\left(\xi_{\mathbf{k}}^{+}, \xi_{\mathbf{k}}^{-}\right)$, where $\xi_{\mathbf{k}}^{ \pm}=\varepsilon_{\mathbf{k}} \pm\left|\mathbf{g}_{\mathbf{k}}\right|$ is the dispersion of the positive-helicity and negative-helicity bands, respectively.

Due to the lack of inversion symmetry, the superconducting gap generally contains an admixture of even-parity spinsinglet and odd-parity spin-triplet pairing states,

$$
\Delta(\mathbf{k})=\left(\psi_{\mathbf{k}} \sigma_{0}+\mathbf{d}_{\mathbf{k}} \cdot \boldsymbol{\sigma}\right)\left(i \sigma_{y}\right),
$$

where $\psi_{\mathbf{k}}$ and $\mathbf{d}_{\mathbf{k}}$ represent the spin-singlet and spin-triplet components, respectively. It is well known that in the absence of interband pairing, the superconducting transition temperature is maximized when the spin-triplet pairing vector $\mathbf{d}_{\mathbf{k}}$ is aligned with the polarization vector $\mathbf{g}_{\mathbf{k}}$ of the SOC ${ }^{42}$ Hence, we parametrize the singlet and triplet components of the superconducting gap function as

$$
\begin{aligned}
& \psi_{\mathbf{k}}=\Delta_{s} f(\mathbf{k})=\frac{r}{r+1} \Delta_{0} f(\mathbf{k}), \\
& \mathbf{d}_{\mathbf{k}}=\Delta_{t} f(\mathbf{k}) \mathbf{l}_{\mathbf{k}}=\frac{1}{r+1} \Delta_{0} f(\mathbf{k}) \mathbf{l}_{\mathbf{k}},
\end{aligned}
$$

where $\mathbf{l}_{\mathbf{k}}=\mathbf{g}_{\mathbf{k}} / \lambda$, with $\lambda$ the SOC strength. Here, $r=$ $\Delta_{s} / \Delta_{t}$ denotes the ratio between the singlet and triplet pairing components. The pairing amplitudes $\Delta_{s}$ and $\Delta_{t}$ are assumed to be positive and constant, i.e., $r \geq 0$. We have included in Eq. (2) a structure factor $f(\mathbf{k})$, which allows us to investigate the effects of higher-orbital-angular-momentum pairing. In the following, we consider the three cases

$$
f(\mathbf{k})= \begin{cases}1 & \text { for }(s+p) \text {-wave, } \\ \left(k_{x}^{2}-k_{y}^{2}\right) / k_{F}^{2} & \text { for }\left(d_{x^{2}-y^{2}}+f\right) \text {-wave, } \\ 2 k_{x} k_{y} / k_{F}^{2} & \text { for }\left(d_{x y}+p\right) \text {-wave }\end{cases}
$$

where $k_{F}$ is the Fermi vector in the absence of spin-orbit interactions. With Eq. (2), it follows that the gaps on the two helicity bands are given by $\Delta_{\mathbf{k}}^{ \pm}=f(\mathbf{k}) \Delta_{0}\left(r \pm\left|\mathbf{l}_{\mathbf{k}}\right|\right) /(r+1)$.

The specific form of the SOC vector $\mathbf{g}_{k}$ (and hence of $l_{k}$ and $\mathbf{d}_{\mathbf{k}}$ ) is constrained by time-reversal symmetry and the crystallographic point-group symmetries of the superconductor. Time-reversal symmetry requires $\mathbf{g}_{\mathbf{k}}$ to be real and an odd function of $\mathbf{k}$. An element $R$ of the crystallographic point group $\mathcal{G}$ of the NCS can be represented as either a proper (for $\operatorname{det} R=+1$ ) or an improper (for $\operatorname{det} R=-1$ ) rotation. Thus, the Bogoliubov-de Gennes Hamiltonian (1) transforms under an operation $R$ of $\mathcal{G}$ as

$$
U_{\tilde{R}} \mathcal{H}\left(R^{-1} \mathbf{k}\right) U_{\tilde{R}}^{\dagger}=+\mathcal{H}(\mathbf{k})
$$

with $\tilde{R}=\operatorname{det}(R) R, U_{\tilde{R}}=\operatorname{diag}\left(u_{\tilde{R}}, u_{\tilde{R}}^{*}\right)$, and $u_{\tilde{R}}$ the spinor representation of $\tilde{R}$, i.e., $u_{\tilde{R}}=\exp [-i(\theta / 2) \hat{\mathbf{n}} \cdot \boldsymbol{\sigma}]$. Here, $\hat{\mathbf{n}}$ denotes the unit vector along the rotation axis of $\tilde{R}$ and $\theta$ is 
the angle of rotation. It follows from Eq. (1) that the lattice symmetries (4) impose the constraint

$$
\mathbf{g}_{\mathbf{k}}=\operatorname{det}(R) R \mathbf{g}_{R^{-1}} \mathbf{k} .
$$

on $\mathbf{g}_{\mathbf{k}}$. To determine the form of $\mathbf{g}_{\mathbf{k}}$, we employ a smallmomentum expansion. Using Eq. (5) we find that for the tetragonal point group $\mathcal{G}=C_{4 v}$ (relevant for $\mathrm{CePt}_{3} \mathrm{Si}$, $\mathrm{CeRhSi}_{3}$, and $\mathrm{CeIrSi}_{3}$ ) the lowest-order symmetry-allowed term is ${ }^{43}$

$$
\mathbf{g}_{\mathbf{k}}=\lambda\left(k_{y} \hat{\mathbf{x}}-k_{x} \hat{\mathbf{y}}\right) .
$$

For the cubic point group $\mathcal{G}=O$ (represented by $\mathrm{Li}_{2} \mathrm{Pd}_{x} \mathrm{Pt}_{3-x} \mathrm{~B}$ and $\mathrm{Mo}_{3} \mathrm{Al}_{2} \mathrm{C}$ ) the vector $\mathrm{g}_{\mathbf{k}}$ takes the form

$$
\begin{aligned}
\mathbf{g}_{\mathbf{k}}= & \lambda\left[k_{x}\left(1+g_{2}\left[k_{y}^{2}+k_{z}^{2}\right]\right) \hat{\mathbf{x}}+k_{y}\left(1+g_{2}\left[k_{x}^{2}+k_{z}^{2}\right]\right) \hat{\mathbf{y}}\right. \\
& \left.+k_{z}\left(1+g_{2}\left[k_{x}^{2}+k_{y}^{2}\right]\right) \hat{\mathbf{z}}\right]
\end{aligned}
$$

with the second-order SOC $g_{2}$. Finally, for the tetrahedral point group $\mathcal{G}=T_{d}$ (relevant for $\mathrm{Y}_{2} \mathrm{C}_{3}$ ) the small-momentum expansion of $\mathbf{g}_{\mathrm{k}}$ yields

$$
\mathbf{g}_{\mathbf{k}}=\lambda\left[k_{x}\left(k_{y}^{2}-k_{z}^{2}\right) \hat{\mathbf{x}}+k_{y}\left(k_{z}^{2}-k_{x}^{2}\right) \hat{\mathbf{y}}+k_{z}\left(k_{x}^{2}-k_{y}^{2}\right) \hat{\mathbf{z}}\right] .
$$

Before deriving the relevant topological invariants for Hamiltonian (1), we first discuss in some detail the discrete symmetries responsible for the nontrivial topological characteristics of $\mathcal{H}(\mathbf{k})$. According to the classification of Refs. 15, 44, and 45, $\mathcal{H}(\mathbf{k})$ belongs to symmetry class DIII since it satisfies two independent antiunitary discrete symmetries: Particle-hole symmetry (PHS) $\mathcal{C}=\mathcal{K} U_{C}$, with $U_{C}=\sigma_{1} \otimes \sigma_{0},{ }^{46}$ and time-reversal symmetry (TRS) $\mathcal{T}=$ $\mathcal{K} U_{T}$, with $U_{T}=\sigma_{0} \otimes i \sigma_{2}$, where $\mathcal{K}$ denotes the complex conjugation operator. TRS acts on the Bogoliubov-de Gennes Hamiltonian $\mathcal{H}(\boldsymbol{k})$ as

$$
U_{T} \mathcal{H}^{\mathrm{T}}(-\mathbf{k}) U_{T}^{\dagger}=+\mathcal{H}(\mathbf{k}),
$$

and PHS as

$$
U_{C} \mathcal{H}^{\mathrm{T}}(-\mathbf{k}) U_{C}^{\dagger}=-\mathcal{H}(\mathbf{k}) .
$$

Combining TRS and PHS, one obtains a third discrete symmetry, the so-called chiral symmetry, which acts as

$$
U_{S}^{\dagger} \mathcal{H}(\mathbf{k}) U_{S}=-\mathcal{H}(\mathbf{k})
$$

with the unitary matrix $U_{S}=i U_{T} U_{C}=-\sigma_{1} \otimes \sigma_{2}$. As we will explain in Sec. [II] it is the chiral symmetry (9) that leads to the protection of the zero-energy surface flat bands. Since $\mathcal{H}(\mathbf{k})$ anticommutes with the unitary matrix $U_{S}$, it can be brought into block off-diagonal form. This is achieved by a unitary transformation that diagonalizes $U_{S}$, e.g., $W U_{S} W^{\dagger}=$ $\operatorname{diag}\left(\sigma_{0},-\sigma_{0}\right)$, with

$$
W=\frac{1}{\sqrt{2}}\left(\begin{array}{cc}
+\sigma_{0} & -\sigma_{2} \\
+i \sigma_{2} & +i \sigma_{0}
\end{array}\right) .
$$

The transformed Hamiltonian reads

$$
\tilde{\mathcal{H}}(\mathbf{k})=W \mathcal{H}(\mathbf{k}) W^{\dagger}=\left(\begin{array}{cc}
0 & D_{\mathbf{k}} \\
D_{\mathbf{k}}^{\dagger} & 0
\end{array}\right),
$$

where the off-diagonal block is given by

$$
D_{\mathbf{k}}=\left(B_{\mathbf{k}} \sigma_{0}+A_{\mathbf{k}} \mathbf{l}_{\mathbf{k}} \cdot \boldsymbol{\sigma}\right)\left(-i \sigma_{2}\right)
$$

with the short-hand notation $A_{\mathbf{k}}=\lambda+i \Delta_{t} f(\mathbf{k})$ and $B_{\mathbf{k}}=$ $\varepsilon_{\mathbf{k}}+i \Delta_{s} f(\mathbf{k})$. We will see in the next section that the topological invariants characterizing the topology properties of $\mathcal{H}(\mathbf{k})$ can be conveniently defined in terms of the off-diagonal block $D_{\mathrm{k}}$, Eq. [10), or its flat-band version. It is found that a crystallographic point-group operation $R$ acts on $D_{\mathbf{k}}$ as $u_{\tilde{R}} D_{R^{-1} \mathbf{k}} u_{\tilde{R}}^{\mathrm{T}}=D_{\mathbf{k}}$, whereas TRS implies $D_{\mathbf{k}}=-D_{-\mathbf{k}}^{\mathrm{T}}$.

Depending on the point group $\mathcal{G}$ and the specific form of $\mathbf{g}_{\mathbf{k}}$, the Hamiltonian $\mathcal{H}(\mathbf{k})$ may in addition to Eq. (7) also satisfy another "time-reversal"-like symmetry that acts only on a two-dimensional plane within the three-dimensional BZ, i.e. a symmetry that acts on $D_{\mathbf{k}}$ as

$$
-D_{-\mathbf{k}_{i}, k_{0}}^{\mathrm{T}}=D_{\mathbf{k}_{i}, k_{0}}
$$

with $\mathbf{k}_{i}=\left(k_{i 1}, k_{i 2}\right)$ the coordinates within the plane $E_{k_{0}}(\hat{\mathbf{u}})=\left\{\mathbf{k} \mid \mathbf{k}=k_{0} \hat{\mathbf{u}}+k_{i 1} \hat{\mathbf{v}}+k_{i 2} \hat{\mathbf{w}}\right\}$ and $k_{0}$ the coordinate perpendicular to $E_{k_{0}}$. Here, $\hat{\mathbf{u}}=\hat{\mathbf{v}} \times \hat{\mathbf{w}}$ and $\hat{\mathbf{v}}$ and $\hat{\mathbf{w}}$ are taken to be orthogonal unit vectors. It follows from Eq. (10c) that a combination of the symmetries (7) and 11a imposes the constraint

$$
\mathbf{g}_{\mathbf{k}_{i}, k_{0}}=\mathbf{g}_{\mathbf{k}_{i},+k_{0}}
$$

on $\mathbf{g}_{\mathbf{k}}=\lambda \mathbf{l}_{\mathbf{k}}$. For example, in the case of the tetragonal point-group $C_{4 v}$ with $\mathbf{g}_{\mathrm{k}}$ given by Eq. (6a), symmetry (11) is satisfied for the one-parameter family of planes $E_{k_{z}}(\hat{\mathbf{z}})$. It is important to note that even though Eq. (11b) has the form of an inversion-type symmetry, it is different from the mirror symmetries imposed by the crystallographic point group, see Eq. (5). We will explain below that the presence of symmetry (11) can give rise to topologically stable arc surface states.

\section{TOPOLOGICAL INVARIANTS IN NODAL NONCENTROSYMMETRIC SUPERCONDUCTORS}

To characterize the topological properties of nodal NCSs in three dimensions, we introduce an integer topological invariant and two $\mathbb{Z}_{2}$ topological numbers. For this purpose it is convenient to work in the off-diagonal basis 10 and to adiabatically deform $\tilde{\mathcal{H}}(\mathbf{k})$ into a flat-band Hamiltonian. The off-diagonal block $D_{\mathbf{k}}$, which is in general non-Hermitian, can through a singular-value decomposition be written as $D_{\mathbf{k}}=U_{\boldsymbol{k}}^{\dagger} \Sigma_{\boldsymbol{k}} V_{\boldsymbol{k}}$, where $U_{\mathbf{k}}$ and $V_{\mathbf{k}}$ are unitary matrices and $\Sigma_{\boldsymbol{k}}$ is a diagonal matrix with the positive eigenenergies

$$
\begin{aligned}
& \Lambda_{1, \mathbf{k}}=\sqrt{\left(\xi_{\mathbf{k}}^{+}\right)^{2}+\left(\Delta_{\mathbf{k}}^{+}\right)^{2}}, \\
& \Lambda_{2, \mathbf{k}}=\sqrt{\left(\xi_{\mathbf{k}}^{-}\right)^{2}+\left(\Delta_{\mathbf{k}}^{-}\right)^{2}}
\end{aligned}
$$

of $\tilde{\mathcal{H}}(\mathbf{k})$ and thus of $\mathcal{H}(\mathbf{k})$ on its diagonal, i.e., $\Sigma_{\boldsymbol{k}}=$ $\operatorname{diag}\left(\Lambda_{1, \mathbf{k}}, \Lambda_{2, \mathbf{k}}\right)$. Assuming that $\Lambda_{1, \mathbf{k}}, \Lambda_{2, \mathbf{k}} \neq 0$, we adiabatically deform the spectrum of $\tilde{\mathcal{H}}(\mathbf{k})$ into flat bands with eigenvalues +1 and -1 , which amounts to replacing $\Sigma_{\mathbf{k}}$ by 
the unit matrix. Hence, the off-diagonal component of the flat-band Hamiltonian is given by ${ }^{9}$

$$
\begin{aligned}
q(\mathbf{k})= & U_{\mathbf{k}}^{\dagger} V_{\mathbf{k}}=\frac{1}{2 \Lambda_{1, \mathbf{k}} \Lambda_{2, \mathbf{k}}}\left[\left\{\Lambda_{\mathbf{k}}^{+} B_{\mathbf{k}}-\Lambda_{\mathbf{k}}^{-} A_{\mathbf{k}}\left|\mathbf{l}_{\mathbf{k}}\right|\right\} \sigma_{0}\right. \\
& \left.+\left\{\Lambda_{\mathbf{k}}^{+} A_{\mathbf{k}}\left|\mathbf{l}_{\mathbf{k}}\right|-\Lambda_{\mathbf{k}}^{-} B_{\mathbf{k}}\right\} \frac{\mathbf{l}_{\mathbf{k}}}{\left|\mathbf{l}_{\mathbf{k}}\right|} \cdot \boldsymbol{\sigma}\right]
\end{aligned}
$$

where $\Lambda_{\mathrm{k}}^{ \pm}=\Lambda_{1, \mathbf{k}} \pm \Lambda_{2, \mathbf{k}}$. As a result of TRS, the $2 \times 2$ unitary matrix $q(\mathbf{k}) \in U(2)$ satisfies $i \sigma_{2} q^{\mathrm{T}}(-\mathbf{k})=q(\mathbf{k}) i \sigma_{2}$.

\section{A. Winding number}

In a three-dimensional nodal superconductor, $q(\mathbf{k})$ is defined only for values of $\mathbf{k}$ for which $\Lambda_{1, \mathbf{k}}, \Lambda_{2, \mathbf{k}} \neq 0$, i.e., for all $\mathrm{k}$ in the BZ except for those on the nodal lines. Hence, the three-dimensional winding number $\nu, \frac{15}{15}$ which characterizes fully gapped systems, is ill-defined for nodal NCSs. However, we can use the one-dimensional winding number $W_{\mathcal{L}}$, which is defined in terms of a one-dimensional momentum-space loop (or line) integral, to characterize the topology of nodal NCSs. To that end, we consider $q(\mathbf{k})$ along a one-dimensional loop (or line) in reciprocal space $\mathbb{R}^{3}$ which does not cross gapless regions. That is, we study the mapping $S^{1} \rightarrow U(2)$ given by $q(\mathbf{k}) \in U(2)$. Since the first homotopy group of $U(2)$ is $\pi_{1}[U(2)]=\mathbb{Z},{ }^{47}$ there is an infinite number of homotopy classes of mappings from $S^{1}$ to $U(2)$, which can be labeled by the one-dimensional winding number

$$
W_{\mathcal{L}}=\frac{1}{2 \pi i} \oint_{\mathcal{L}} d k_{l} \operatorname{Tr}\left[q^{\dagger}(\mathbf{k}) \partial_{k_{l}} q(\mathbf{k})\right] .
$$

The integral is to be evaluated along the path $\mathcal{L}$ parametrized by $k_{l}$. We observe that $W_{\mathcal{L}}$ is quantized for any closed loop $\mathcal{L}$ that does not intersect with nodal lines. If $\mathcal{L}$ encircles a line node, $W_{\mathcal{L}}$ determines the topological charge and hence the topological stability of the nodal line. Using Eq. (13) we find

$$
\begin{aligned}
& W_{\mathcal{L}}=\frac{1}{2 \pi i} \oint_{\mathcal{L}} d k_{l} \partial_{k_{l}}[\ln \operatorname{det} q(\mathbf{k})] \\
& =\frac{1}{2 \pi} \oint_{\mathcal{L}} d k_{l} \partial_{k_{l}}\left[\arg \left(B_{\mathbf{k}}^{2}-A_{\mathbf{k}}^{2}\left|\mathbf{l}_{\mathbf{k}}\right|^{2}\right)\right] \\
& =\frac{1}{2 \pi} \oint_{\mathcal{L}} d k_{l} \partial_{k_{l}}\left[\arg \left(\xi_{\mathbf{k}}^{+}+i \Delta_{\mathbf{k}}^{+}\right)+\arg \left(\xi_{\mathbf{k}}^{-}+i \Delta_{\mathbf{k}}^{-}\right)\right],
\end{aligned}
$$

where, in going from the first to the second line, we have used the identity $|\operatorname{det} q(\mathbf{k})|=1$.

Assuming that the energy scales of the gap functions are much smaller than those of the normal-state dispersions (i.e, for a weak-pairing superconductor), we can rescale $\Delta_{\mathbf{k}}^{ \pm}$without changing $W_{\mathcal{L}}$, such that the gaps are nonzero only within a small neighborhood of the Fermi surface sheets [cf. Eq. [12]]. In this limit we find that the phase $\arg \left(\xi_{\mathbf{k}}^{\nu}+i \Delta_{\mathbf{k}}^{\nu}\right)$ is constant far away from the Fermi surface of helicity $\nu= \pm$ and that it jumps by

$$
-\pi \operatorname{sgn}\left(\left.\partial_{k_{l}} \xi_{\mathbf{k}}^{\nu}\right|_{\mathbf{k}=\mathbf{k}_{F}^{0}}\right) \operatorname{sgn}\left(\Delta_{\mathbf{k}_{F}^{0}}^{\nu}\right)
$$

where the path $\mathcal{L}$ crosses the Fermi surface at $\mathbf{k}_{F}^{0}$. It follows that the winding number $W_{\mathcal{L}}$ can be simplified to (cf. Ref.11)

$$
W_{\mathcal{L}}=-\frac{1}{2} \sum_{\nu= \pm} \sum_{\mathbf{k}_{F}^{0} \in S_{\mathcal{L}}^{\nu}} \operatorname{sgn}\left(\left.\partial_{k_{l}} \xi_{\mathbf{k}}^{\nu}\right|_{\mathbf{k}=\mathbf{k}_{F}^{0}}\right) \operatorname{sgn}\left(\Delta_{\mathbf{k}_{F}^{0}}^{\nu}\right)
$$

where the set of points $S_{\mathcal{L}}^{\nu}$ is given by the intersection of the path $\mathcal{L}$ with the Fermi surface for helicity $\nu$. Thus Eq. (17) shows that for a weak-pairing NCS $W_{\mathcal{L}}$ is completely determined by the phase structure of the superconducting gaps $\Delta_{\mathbf{k}}^{+}$ and $\Delta_{\mathrm{k}}^{-}$in the vicinity of the positive-helicity and negativehelicity Fermi surfaces, respectively. Moreover, it follows from Eq. (17) that nodal lines necessarily carry nontrivial topological charge $W_{\mathcal{L}} \neq 0$, irrespective of the particular form of the band structure or the crystal point-group symmetries.

\section{B. Two-dimensional $\mathbb{Z}_{2}$ topological invariant}

The two-dimensional $\mathbb{Z}_{2}$ topological invariant introduced in this subsection is defined only for NCSs satisfying symmetry (11). We therefore consider a Hamiltonian $\mathcal{H}(\mathbf{k})$ of the form (1) that is invariant under symmetry (11) for the plane $E_{k_{0}}(\hat{\mathbf{u}})$. Furthermore, we assume that the nodal lines of $\mathcal{H}(\mathbf{k})$ do not cross $E_{k_{0}}(\hat{\mathbf{u}})$. Hence, $\mathcal{H}(\mathbf{k})$ restricted to the plane $E_{k_{0}}(\hat{\mathbf{u}})$ can be regarded as describing a two-dimensional fully gapped superconductor invariant under both TRS [i.e., symmetry [11] and PHS [i.e., the combination of chiral symmetry and symmetry [11]]. Such a two-dimensional system belongs to symmetry class DIII and its topological characteristics are described by the $\mathbb{Z}_{2}$ topological invariant $\frac{8|9| 17|21|}{23}$

$$
\begin{aligned}
N_{E_{k_{0}}}^{2 \mathrm{D}}= & \prod_{k_{i 2}=0, \pi} \frac{\operatorname{Pf}\left[i \sigma_{2} \hat{q}\left(\pi, k_{i 2}, k_{0}\right)\right]}{\operatorname{Pf}\left[i \sigma_{2} \hat{q}\left(0, k_{i 2}, k_{0}\right)\right]} \\
& \times e^{-\frac{1}{2} \int_{0}^{\pi} d k_{i 1} \operatorname{Tr}\left[\hat{q}^{\dagger}(\mathbf{k}) \partial_{k_{i 1}} \hat{q}(\mathbf{k})\right]},
\end{aligned}
$$

where $\hat{q}(\mathbf{k})$ represents a lattice regularization of $q(\mathbf{k})^{48}$ and $N_{E_{k_{0}}}^{2 \mathrm{D}}=-1(+1)$ indicates a topologically nontrivial (trivial) character. In Eq. (18), we have assumed that the coordinates $\left(k_{i 1}, k_{i 2}\right)$ within the plane $E_{k_{0}}(\hat{\mathbf{u}})$ are chosen such that $\mathbf{K}_{i}=(0,0),(\pi, 0),(0, \pi)$, and $(\pi, \pi)$ are left invariant under symmetry (11). Note that at these points $i \sigma_{2} \hat{q}\left(\mathbf{K}_{i}, k_{0}\right)$ is antisymmetric, so that the Pfaffian is well-defined.

As before, we consider the weak-pairing limit and set the gaps $\Delta_{\mathrm{k}}^{ \pm}$to zero far away from the Fermi surfaces. Using Eq. (17), we find that in this approximation the exponential factor in Eq. (18) reduces to

$$
\prod_{\nu= \pm} \prod_{\mathbf{k}_{F}^{0} \in \tilde{S}_{E_{k_{0}}^{\nu}}^{\nu}} i \operatorname{sgn}\left(\left.\Delta_{\mathbf{k}_{F}^{0}}^{\nu} \partial_{k_{i 1}} \xi_{\mathbf{k}}^{\nu}\right|_{\mathbf{k}=\mathbf{k}_{F}^{0}}\right)
$$

where the set of points $\tilde{S}_{E_{k_{0}}}^{\nu}$ is given by the intersection of the Fermi surface for helicity $\nu$ with the integration paths $\mathbf{k}_{i}:(0,0) \rightarrow(\pi, 0)$ and $\mathbf{k}_{i}:(0, \pi) \rightarrow(\pi, \pi)$. Assuming that the Fermi level does not cross the positive-helicity or negativehelicity bands at $\left(\mathbf{K}_{i}, k_{0}\right)$, we find that $\operatorname{Pf}\left[i \sigma_{2} \hat{q}\left(\mathbf{K}_{i}, k_{0}\right)\right]$ is either +1 or -1 depending on whether the helicity bands at 
$\left(\mathbf{K}_{i}, k_{0}\right)$ are occupied or unoccupied. As a result, the $\mathbb{Z}_{2}$ invariant for $\mathcal{H}(\mathbf{k})$ simplifies to

$$
N_{E_{k_{0}}}^{2 \mathrm{D}}=\operatorname{sgn}\left(\Delta_{E_{k_{0}}}^{+}\right) \operatorname{sgn}\left(\Delta_{E_{k_{0}}}^{-}\right),
$$

where $\operatorname{sgn}\left(\Delta_{E_{k_{0}}}^{ \pm}\right)$denotes the sign of the gap on the Fermi line given by the intersection of $E_{k_{0}}(\hat{\mathbf{u}})$ with the positive/negativehelicity Fermi surface. Observe that Eq. (20) does not depend on the lattice regularization and hence is valid also in the continuum limit.

For a NCS that is invariant under symmetry (11) for a oneparameter family of planes $E_{k}(\hat{\mathbf{u}})$, with for example $k \in \mathbb{R}$, it is possible to assign a $\mathbb{Z}_{2}$ topological charge to the line nodes, provided that there are fully gapped regions in momentum space separating different line nodes. For concreteness, let us consider the situation where a nodal line is located within the plane $E_{k=k_{0}}(\hat{\mathbf{u}})$. The $\mathbb{Z}_{2}$ topological charge of this nodal ring can be defined as

$$
\tilde{N}^{2 \mathrm{D}}=N_{E_{k_{0}^{+}}^{+}}^{2 \mathrm{D}} N_{E_{k_{0}^{-}}}^{2 \mathrm{D}},
$$

where $E_{k_{0}^{+}}$and $E_{k_{0}^{-}}$represent two planes that are located on either side of the nodal line. For the tetragonal pointgroup $C_{4 v}$ [with $\mathbf{g}_{\mathbf{k}}$ of the form 6a ] ], the two-dimensional $\mathbb{Z}_{2}$ number can be defined for the one-parameter family of planes $E_{k_{z}}(\hat{\mathbf{z}})$. The nodal rings, which are centered around the $k_{z}$-axis, consequently carry a nontrivial $\mathbb{Z}_{2}$ topological charge $\tilde{N}_{\alpha}^{2 \mathrm{D}}=N_{E_{k_{z}=0}}^{2 \mathrm{D}} N_{E_{k_{z}=\alpha k_{F}}}^{2 \mathrm{D}}$, where $k_{F}=\sqrt{2 m \mu / \hbar^{2}}$ and $\alpha= \pm$ distinguishes between the topological charges of the nodal rings in the upper $\left(k_{z}>0\right)$ and lower $\left(k_{z}<0\right)$ half-spaces.

\section{One-dimensional $\mathbb{Z}_{2}$ topological invariant}

Finally, we also introduce a one-dimensional $\mathbb{Z}_{2}$ invariant that characterizes the topological properties of $\mathcal{H}(\mathbf{k})$ restricted to a time-reversal-invariant loop (or line) $\mathcal{L}$, which is mapped onto itself under $\mathbf{k} \rightarrow-\mathbf{k}$. Similarly to Eq. (18), the one-dimensional $\mathbb{Z}_{2}$ topological number can be conveniently defined in terms of the lattice-regularized version of $q(\mathbf{k})$, Eq. $133,, 914121123$

$$
N_{\mathcal{L}}^{1 \mathrm{D}}=\frac{\operatorname{Pf}\left[i \sigma_{2} \hat{q}\left(\mathbf{K}_{2}\right)\right]}{\operatorname{Pf}\left[i \sigma_{2} \hat{q}\left(\mathbf{K}_{1}\right)\right]} e^{-\frac{1}{2} \oint_{\mathcal{L}} d k_{l} \operatorname{Tr}\left[\hat{q}^{\dagger}(\mathbf{k}) \partial_{k_{l}} \hat{q}(\mathbf{k})\right]},
$$

where we have assumed that $\mathcal{L}$ does not cross the nodal lines and $\mathbf{K}_{1}$ and $\mathbf{K}_{2}$ denote the two time-reversal-invariant momenta on the path $\mathcal{L}$. Repeating similar steps as in the previous subsection, we find that for a weak-pairing superconductor $N_{\mathcal{L}}^{1 \mathrm{D}}$ simplifies to

$$
N_{\mathcal{L}}^{1 \mathrm{D}}=\operatorname{sgn}\left(\Delta_{\mathcal{L}}^{+}\right) \operatorname{sgn}\left(\Delta_{\mathcal{L}}^{-}\right)
$$

where $\operatorname{sgn}\left(\Delta_{\mathcal{L}}^{ \pm}\right)$represents the sign of the gap at the points given by the intersection of $\mathcal{L}$ with the positive/negativehelicity Fermi surface.

\section{Topological criteria for the existence of zero-energy surface states}

As a consequence of a bulk-boundary correspondence, 15449 a nontrivial value of any of the three topological invariants (14), (18), and (22) signals the appearance of zero-energy states at the surface of the NCS. That is, $W_{\mathcal{L}} \neq 0$ leads to surface flat bands, $\tilde{N}_{ \pm}^{2 \mathrm{D}}=-1$ gives rise to arc surface states, and $N_{\mathcal{L}}^{1 \mathrm{D}}=-1$ results in Majorana modes at time-reversalinvariant momenta of the surface BZ. In the following, we discuss in detail the topological criteria for the existence of these three types of surface states. For that purpose, we denote the coordinates parallel (perpendicular) to a given surface of the NCS by $\mathbf{r}_{\|}\left(r_{\perp}\right)$ and the corresponding momenta by $\mathbf{k}_{\|}\left(k_{\perp}\right)$.

a. Surface flat bands: The appearance of surface flat bands can be understood by considering a continuous deformation of the closed integration path $\mathcal{L}$ of $W_{\mathcal{L}}$, Eq. (14), into an infinite semicircle, such that the diameter of the semicircle contains the line $\left(k_{\perp}, \mathbf{k}_{\|}\right)$, with $k_{\perp} \in \mathbb{R}$ and $\mathbf{k}_{\|}$fixed. This path deformation does not alter the value of the integral, as long as no nodal line is crossed while deforming $\mathcal{L}$; if a nodal line is crossed $W_{\mathcal{L}}$ changes by \pm 1 . As can be seen from Eq. (17), the integral along the arc of the semicircle is zero and hence the integral along the diameter is given by ${ }^{50}$

$$
W_{(l m n)}\left(\mathbf{k}_{\|}\right)=-\frac{1}{2} \sum_{\nu= \pm}\left[\operatorname{sgn}\left(\Delta_{\mathbf{k}_{F, \nu}}^{\nu}\right)-\operatorname{sgn}\left(\Delta_{\widetilde{\mathbf{k}}_{F, \nu}}^{\nu}\right)\right]
$$

where $\mathbf{k}_{F, \pm}=\left(k_{\perp, \pm}, \mathbf{k}_{\|}\right)$and $\widetilde{\mathbf{k}}_{F, \pm}=\left(\widetilde{k}_{\perp, \pm}, \mathbf{k}_{\|}\right)$satisfy $\xi_{\mathbf{k}_{F, \pm}}^{ \pm}=0$ and $\xi_{\widetilde{\mathbf{k}}_{F, \pm}}^{ \pm}=0$, respectively, with $\mathbf{k}_{F, \pm}\left(\widetilde{\mathbf{k}}_{F, \pm}\right)$ corresponding to solutions with positive (negative) signs of the Fermi-velocity component perpendicular to the surface. The subscript $(l m n)$ in Eq. (24) parametrizes the direction perpendicular to the surface. From Eq. 24] it follows that zeroenergy surface states occur whenever $W_{(l m n)}\left(\mathbf{k}_{\|}\right) \neq 0$, which corresponds to regions of the surface $\mathrm{BZ}$ that are bounded by the projections of the nodal rings of the bulk gap.

b. Arc surface states: Consider an NCS satisfying symmetry (11) for $E_{k}(\hat{\mathbf{u}})$, with $k \in \mathbb{R}$, and with two nodal rings carrying nontrivial $\mathbb{Z}_{2}$ topological charge, Eq. 21]. Any twodimensional subsystem $E_{k}(\hat{\mathbf{u}})$ lying between these two line nodes can be viewed as a time-reversal-invariant topological superconductor in class DIII. When these two-dimensional subsystems are terminated by a boundary, there appear helical Majorana edge states. These Majorana modes cross zero energy on the surface BZ, somewhere in between the projections of the two nodal rings. Thus, there is a one-dimensional arc of zero-energy states connecting the projections of the two nodal rings. If the projected nodal rings have a finite overlap in the surface BZ, cancellation occurs and no arc surface states are expected. By symmetry, the arc surface states always appear at those surface momenta that are invariant under symmetry (11). It is interesting to note that these arc surface states are reminiscent of the Fermi arcs that appear at the surface of Weyl semimetals, which have recently been discussed in the context of the A phase of ${ }^{3} \mathrm{He}, \frac{51}{53}$ pyrochlore iridates, $\stackrel{52}{ }$ and topological insulator multilayers 53 
c. Majorana surface states: We observe that the gap $\Delta_{\mathbf{k}}^{\nu}$ (with $\nu= \pm$ ) has the same values at any two momenta related by TRS. Thus, for a given time-reversal-invariant surface momentum $\mathbf{K}_{\|}$, the one-dimensional $\mathbb{Z}_{2}$ number 23 reads

$$
N_{\mathbf{K}_{\|}}^{1 \mathrm{D}}=\operatorname{sgn}\left(\Delta_{\mathbf{K}_{F,+}}^{+}\right) \operatorname{sgn}\left(\Delta_{\mathbf{K}_{F,-}}^{-}\right),
$$

where $\mathbf{K}_{F, \pm}=\left(K_{\perp \pm}, \mathbf{K}_{\|}\right)$satisfies $\xi_{\mathbf{K}_{F, \pm}^{ \pm}}^{ \pm}=0$ and the sign of the Fermi-velocity component $\left.\partial_{K_{\perp}} \xi_{\mathbf{k}}^{ \pm}\right|_{\mathbf{k}=\mathbf{K}_{F, \pm}}$ is assumed to be positive. Consequently, $N_{\mathbf{K}_{\|}}^{1 \mathrm{D}}=-1$ indicates the presence of Kramers-degenerate Majorana surface modes at the time-reversal-invariant momentum $\mathbf{K}_{\|}$of the surface BZ.

Before closing this subsection, we note that the topological protection of boundary modes in nodal NCSs is weaker than the protection of surface states in strong topological insulators or superconductors with a full gap in the bulk. Nevertheless, the surface states in nodal NCSs are expected to possess a certain robustness against disorder, similar to the zero-energy edge states in graphene $e^{54}$ or in $\mathrm{d}_{x^{2}-y^{2}}$-wave superconductors ${ }^{55}$ For example, we expect that weak surface roughness will result in a broadening of the surface states described above, but the surface spectral function will still display sharp peaks at the bound state energies. Deeper examination of the effect of disorder is beyond the scope of this paper, and is left to later publications.

\section{BOUND STATE SPECTRA}

In this section, we use quasiclassical scattering theory to derive conditions for the existence of surface bound states. We will see that the quasiclassical criteria for the appearance of zero-energy surface states are in perfect agreement with the topological criteria given in the previous section. Hence, this provides a verification of the bulk-boundary correspondence that relates nontrivial topological characteristics of quasiparticle wavefunctions in the bulk to the existence of zero-energy states at the surface.

\section{A. General condition for bound states}

The wavevector component $\mathbf{k}_{\|}$parallel to an ideal surface is a good quantum number due to translational invariance, and so we can construct the bound-state wavefunction for each point in the surface $\mathrm{BZ}$ independently. For a given $\mathbf{k}_{\|}$, the usual quasiclassical method $\sqrt{56}$ is only applicable if there are at most two solutions in each helicity sector. If there are more than two solutions in either sector (for example for a concave Fermi surface) we are unable to uniquely determine the coefficients of the different spinors appearing in the bound-statewavefunction ansatz for the given $\mathbf{k}_{\|}$. Our approach is therefore reasonable in the limit of weak to moderate SOC, where the concavity of the Fermi surface is only relevant over a small fraction of the surface BZ.

For given $\mathbf{k}_{\|}$we must therefore in general consider four wavevectors $\mathbf{k}_{ \pm}=\left(k_{\perp, \pm}, \mathbf{k}_{\|}\right)$and $\widetilde{\mathbf{k}}_{ \pm}=\left(\widetilde{k}_{\perp, \pm}, \mathbf{k}_{\|}\right)$, which satisfy $\xi_{\mathbf{k}_{ \pm}}^{ \pm}=\xi_{\tilde{\mathbf{k}}_{ \pm}}^{ \pm}=0$. We classify a solution as propagating if $k_{\perp}$ is real and as evanescent otherwise. In the former case, $\mathbf{k}_{ \pm}$and $\widetilde{\mathbf{k}}_{ \pm}$correspond to solutions with opposite signs of the Fermi-velocity component perpendicular to the surface. We assume that the NSC is located in the half-space $r_{\perp}>0$.

\section{Propagating solutions on both Fermi surfaces}

If there are propagating solutions on both the positivehelicity and the negative-helicity Fermi surfaces we have the wavefunction ansatz

$$
\begin{aligned}
\Psi\left(\mathbf{k}_{\|} ; \mathbf{r}\right) & =\sum_{\nu= \pm} \Psi_{\nu}\left(\mathbf{k}_{\|} ; \mathbf{r}\right) e^{i \mathbf{k}_{\|} \cdot \mathbf{r}}, \\
\Psi_{\nu}\left(\mathbf{k}_{\|} ; \mathbf{r}\right) & =\sum_{\mathbf{k}=\mathbf{k}_{\nu}, \widetilde{\mathbf{k}}_{\nu}} \alpha_{\nu}(\mathbf{k}) \psi_{\nu}(\mathbf{k}) e^{i k_{\perp} r_{\perp}} e^{-\kappa_{\mathbf{k}}^{\nu} r_{\perp}},
\end{aligned}
$$

where the positive-helicity and negative-helicity spinors are given by

$$
\begin{aligned}
& \psi_{+}(\mathbf{k})=\left(1, \frac{l_{\mathbf{k}}^{x}+i l_{\mathbf{k}}^{y}}{\left|\mathbf{l}_{\mathbf{k}}\right|+l_{\mathbf{k}}^{x}},-\frac{l_{\mathbf{k}}^{x}+i l_{\mathbf{k}}^{y}}{\left|\mathbf{l}_{\mathbf{k}}\right|+l_{\mathbf{k}}^{x}} \gamma_{\mathbf{k}}^{+}, \gamma_{\mathbf{k}}^{+}\right)^{T}, \\
& \psi_{-}(\mathbf{k})=\left(\frac{l_{\mathbf{k}}^{x}-i l_{\mathbf{k}}^{y}}{\left|I_{\mathbf{k}}\right|+l_{\mathbf{k}}^{x}},-1, \gamma_{\mathbf{k}}^{-}, \frac{l_{\mathbf{k}}^{x}-i l_{\mathbf{k}}^{y}}{\left|\mathbf{l}_{\mathbf{k}}\right|+l_{\mathbf{k}}^{x}} \gamma_{\mathbf{k}}^{-}\right)^{T},
\end{aligned}
$$

respectively, with

$$
\begin{aligned}
& \gamma_{\mathbf{k}}^{ \pm}=\frac{1}{\Delta_{\mathbf{k}}^{ \pm}}\left[E-i \operatorname{sgn}\left(v_{F, \perp}^{ \pm}(\mathbf{k})\right) \sqrt{\left|\Delta_{\mathbf{k}}^{ \pm}\right|^{2}-E^{2}}\right], \\
& \kappa_{\mathbf{k}}^{ \pm}=\frac{1}{\hbar\left|v_{F, \perp}^{ \pm}(\mathbf{k})\right|} \sqrt{\left|\Delta_{\mathbf{k}}^{ \pm}\right|^{2}-E^{2}},
\end{aligned}
$$

and $v_{F, \perp}^{\nu}(\mathbf{k})$ is the component of the $\nu= \pm$ helicity Fermi velocity normal to the surface.

A bound state is realized when it is possible to choose the coefficients $\alpha_{\nu}(\mathbf{k})$ in Eq. 26) such that the wavefunction vanishes at the surface, i.e., $\left.\Psi\left(\mathbf{k}_{\|} ; \mathbf{r}\right)\right|_{r_{\perp}=0}=0$. This is equivalent to the condition

$$
\begin{aligned}
0= & \left(\gamma_{\mathbf{k}_{-}}^{-}-\gamma_{\widetilde{\mathbf{k}}_{-}}^{\bar{y}}\right)\left(\gamma_{\widetilde{\mathbf{k}}_{+}}^{+}-\gamma_{\mathbf{k}_{+}}^{+}\right)\left[\left(\left|\mathbf{l}_{\mathbf{k}_{+}}\right|+l_{\mathbf{k}_{+}}^{z}\right)\left(\left|\mathbf{l}_{\widetilde{\mathbf{k}}_{-}}\right|+l_{\widetilde{\mathbf{k}}_{-}}^{z}\right)-\left(l_{\mathbf{k}_{+}}^{x}+i l_{\mathbf{k}_{+}}^{y}\right)\left(-l_{\widetilde{\mathbf{k}}_{-}}^{x}+i l_{\widetilde{\mathbf{k}}_{-}}^{y}\right)\right] \\
& \times\left[\left(\left|\mathbf{l}_{\mathbf{k}_{-}}\right|+l_{\mathbf{k}_{-}}^{z}\right)\left(\left|\mathbf{l}_{\widetilde{\mathbf{k}}_{+}}\right|+l_{\mathbf{\mathbf { k }}_{+}}^{z}\right)-\left(-l_{\mathbf{k}_{-}}^{x}+i l_{\mathbf{k}_{-}}^{y}\right)\left(l_{\widetilde{\mathbf{k}}_{+}}^{x}+i l_{\widetilde{\mathbf{k}}_{+}}^{y}\right)\right] \\
& +\left(\gamma_{\mathbf{k}_{-}}^{-}-\gamma_{\widetilde{\mathbf{k}}_{-}}^{+}\right)\left(\gamma_{\mathbf{k}_{+}}^{+}-\gamma_{\widetilde{\mathbf{k}}_{-}}^{-}\right)\left[\left(\left|\mathbf{l}_{\mathbf{k}_{+}}\right|+l_{\mathbf{k}_{+}}^{z}\right)\left(l_{\widetilde{\mathbf{k}}_{+}}^{x}+i l_{\widetilde{\mathbf{k}}_{+}}^{y}\right)-\left(l_{\mathbf{k}_{+}}^{x}+i l_{\mathbf{k}_{+}}^{y}\right)\left(\left|\mathbf{l}_{\widetilde{\mathbf{k}}_{+}}\right|+l_{\widetilde{\mathbf{k}}_{+}}^{z}\right)\right] \\
& \times\left[\left(\left|\mathbf{l}_{\mathbf{k}_{-}}\right|+l_{\mathbf{k}_{-}}^{z}\right)\left(-l_{\widetilde{\mathbf{k}}_{-}}^{x}+i l_{\widetilde{\mathbf{k}}_{-}}^{y}\right)-\left(-l_{\mathbf{k}_{-}}^{x}+i l_{\mathbf{k}_{-}}^{y}\right)\left(\left|\mathbf{l}_{\widetilde{\mathbf{k}}_{-}}\right|+l_{\widetilde{\mathbf{k}}_{-}}^{z}\right)\right]
\end{aligned}
$$


Solutions of this equation satisfying $|E|<$ $\min \left\{\left|\Delta_{\mathbf{k}_{ \pm}}^{ \pm}\right|,\left|\Delta_{\widetilde{\mathbf{k}}_{ \pm}}^{ \pm}\right|\right\}$are the bound-state energies. Focusing on zero-energy solutions that occur within a finite region of the surface BZ, we find three possibilities for such states:

(i) $\operatorname{sgn}\left(\Delta_{\mathbf{k}_{-}}^{-} \Delta_{\widetilde{\mathbf{k}}_{-}}^{-}\right)=-1$ and $\operatorname{sgn}\left(\Delta_{\mathbf{k}_{+}}^{+} \Delta_{\widetilde{\mathbf{k}}_{+}}^{+}\right)=+1$,

(ii) $\operatorname{sgn}\left(\Delta_{\mathbf{k}_{-}}^{-} \Delta_{\widetilde{\mathbf{k}}_{-}}^{-}\right)=+1$ and $\operatorname{sgn}\left(\Delta_{\mathbf{k}_{+}}^{+} \Delta_{\widetilde{\mathbf{k}}_{+}}^{+}\right)=-1$,

(iii) $\operatorname{sgn}\left(\Delta_{\mathbf{k}_{-}}^{-} \Delta_{\tilde{\mathbf{k}}_{-}}^{-}\right)=-1$ and $\operatorname{sgn}\left(\Delta_{\mathbf{k}_{+}}^{+} \Delta_{\tilde{\mathbf{k}}_{+}}^{+}\right)=-1$ and $\operatorname{sgn}\left(\Delta_{\mathbf{k}_{-}}^{-} \Delta_{\widetilde{\mathbf{k}}_{+}}^{+}\right)=-1$.

These conditions agree perfectly with the topological criterion (24). That is, scenarios (i) and (ii) correspond to topologically protected singly degenerate zero-energy states with winding number $W_{(l m n)}= \pm 1$, while (iii) gives doubly degenerate states of winding number $W_{(\operatorname{lmn})}= \pm 2$. We note that a careful examination of the terms involving $l_{k}$ can also yield zero-energy dispersing states at isolated points or in a line. These states include, but are not limited to, the topologically protected Majorana modes and arc surface states introduced in Sec. IIID The general form of this condition is rather cumbersome, but it simplifies significantly in the limit of weak SOC and so we defer discussion to this case.

\section{Propagating solutions on only one Fermi surface}

In the case that there are propagating solutions only on the negative-helicity Fermi surface, the negative-helicity components of the wavefunction ansatz remain as above, but the positive-helicity components are now written

$$
\begin{aligned}
\Psi_{+}\left(\mathbf{k}_{\|} ; \mathbf{r}\right)= & \left\{\alpha_{+}(\mathbf{p}) \phi(\mathbf{p}) e^{-i q_{\mathbf{p}} r_{\perp}}\right. \\
& \left.+\widetilde{\alpha}_{+}(\mathbf{p}) \widetilde{\phi}(\mathbf{p}) e^{i q_{\mathbf{p}} r_{\perp}}\right\} e^{i p_{\perp} r_{\perp}},
\end{aligned}
$$

where $\mathbf{p}$ is chosen from $\mathbf{k}_{+}$and $\widetilde{\mathbf{k}}_{+}$such that the imaginary part of $p_{\perp}$ is positive, the spinors $\phi(\mathbf{p})$ and $\widetilde{\phi}(\mathbf{p})$ are defined by

$$
\begin{aligned}
& \phi(\mathbf{p})=\left(1, \frac{l_{\mathbf{p}}^{x}+i l_{\mathbf{p}}^{y}}{\left|\mathbf{l}_{\mathbf{p}}\right|+l_{\mathbf{p}}^{z}},-\frac{l_{\mathbf{p}}^{x}+i l_{\mathbf{p}}^{y}}{\left|\mathbf{l}_{\mathbf{p}}\right|+l_{\mathbf{p}}^{z}} \Gamma_{\mathbf{p}}, \Gamma_{\mathbf{p}}\right)^{T}, \\
& \widetilde{\phi}(\mathbf{p})=\left(1, \frac{l_{\mathbf{p}}^{x}+i l_{\mathbf{p}}^{y}}{\left|\mathbf{l}_{\mathbf{p}}\right|+l_{\mathbf{p}}^{z}},-\frac{l_{\mathbf{p}}^{x}+i l_{\mathbf{p}}^{y}}{\left|\mathbf{l}_{\mathbf{p}}\right|+l_{\mathbf{p}}^{z}} \widetilde{\Gamma}_{\mathbf{p}}, \widetilde{\Gamma}_{\mathbf{p}}\right)^{T},
\end{aligned}
$$

and we have

$$
\begin{aligned}
& \Gamma_{\mathbf{p}}=\frac{1}{\Delta_{\mathbf{p}}^{+}}\left(E+i \sqrt{\left(\Delta_{\mathbf{p}}^{+}\right)^{2}-E^{2}}\right), \\
& \widetilde{\Gamma}_{\mathbf{p}}=\frac{1}{\Delta_{\mathbf{p}}^{+}}\left(E-i \sqrt{\left(\Delta_{\mathbf{p}}^{+}\right)^{2}-E^{2}}\right), \\
& q_{\mathbf{p}}=\frac{1}{\hbar \operatorname{Im}\left\{v_{F, \perp}^{+}(\mathbf{p})\right\}} \sqrt{\left(\Delta_{\mathbf{p}}^{+}\right)^{2}-E^{2}} .
\end{aligned}
$$

Using the same argument as above, we find the condition

$$
\begin{aligned}
0= & \left(\widetilde{\Gamma}_{\mathbf{p}}-\Gamma_{\mathbf{p}}\right)\left(\gamma_{\mathbf{k}_{-}}^{-}-\gamma_{\widetilde{\mathbf{k}}_{-}}^{-}\right)\left[\left(\left|\mathbf{l}_{\mathbf{p}}\right|+l_{\mathbf{p}}^{z}\right)\left(\left|\mathbf{l}_{\mathbf{k}_{-}}\right|+l_{\mathbf{k}_{-}}^{z}\right)-\left(l_{\mathbf{p}}^{x}+i l_{\mathbf{p}}^{y}\right)\left(-l_{\mathbf{k}_{-}}^{x}+i l_{\mathbf{k}_{-}}^{y}\right)\right] \\
& \times\left[\left(\left|\mathbf{l}_{\mathbf{p}}\right|+l_{\mathbf{p}}^{z}\right)\left(\left|\mathbf{l}_{\widetilde{\mathbf{k}}_{-}}\right|+l_{\widetilde{\mathbf{k}}_{-}}^{z}\right)-\left(l_{\mathbf{p}}^{x}+i l_{\mathbf{p}}^{y}\right)\left(-l_{\widetilde{\mathbf{k}}_{-}}^{x}+i l_{\widetilde{\mathbf{k}}_{-}}^{y}\right)\right]
\end{aligned}
$$

for the formation of bound states. Unlike Eq. 27], this condition only allows for the existence of singly degenerate zeroenergy surface states which occur whenever $\operatorname{sgn}\left(\Delta_{\mathbf{k}_{-}}^{-}\right)=$ $-\operatorname{sgn}\left(\Delta_{\tilde{\mathbf{k}}_{-}}^{\overline{-}_{-}}\right) !{ }^{[57}$ Again, we find that this criterion matches with the topological one, Eq. 24).

\section{Limit of weak spin-orbit coupling}

Because of the difficulty of working with spin-orbit split Fermi surfaces, many theoretical studies assume that the SOC is weak so that the spin splitting of the Fermi surfaces can be ignored. ${ }^{102025}$ 28 from Eq. 27 by setting $\mathbf{k}_{+}=\mathbf{k}_{-}=\mathbf{k}$ and $\widetilde{\mathbf{k}}_{+}=\widetilde{\mathbf{k}}_{-}=\widetilde{\mathbf{k}}$ to yield the compact bound-state condition

$$
\begin{aligned}
0= & \left(\gamma_{\widetilde{\mathbf{k}}}^{+}-\gamma_{\mathbf{k}}^{-}\right)\left(\gamma_{\widetilde{\mathbf{k}}}^{-}-\gamma_{\mathbf{k}}^{+}\right)\left(\left|\mathbf{l}_{\mathbf{k}}\right|\left|\mathbf{l}_{\widetilde{\mathbf{k}}}\right|-\mathbf{l}_{\mathbf{k}} \cdot \mathbf{l}_{\widetilde{\mathbf{k}}}\right) \\
& +\left(\gamma_{\widetilde{\mathbf{k}}}^{-}-\gamma_{\mathbf{k}}^{-}\right)\left(\gamma_{\widetilde{\mathbf{k}}}^{+}-\gamma_{\mathbf{k}}^{+}\right)\left(\left|\mathbf{l}_{\mathbf{k}}\right|\left|\mathbf{l}_{\widetilde{\mathbf{k}}}\right|+\mathbf{l}_{\mathbf{k}} \cdot \mathbf{l}_{\widetilde{\mathbf{k}}}\right)
\end{aligned}
$$

This result was previously presented in Ref. 10 and is utilized here to obtain the surface bound-state spectra for the $O$ and $T_{d}$ point groups. Although describing a physically idealized situation, Eq. (30) is useful as it significantly simplifies the discussion of the zero-energy dispersing states. In particular, we find that such states are possible if (i) $\mathbf{l}_{\mathbf{k}} \cdot \mathbf{l}_{\widetilde{\mathbf{k}}}=\left|\mathbf{l}_{\mathbf{k}}\right|\left|\mathbf{l}_{\widetilde{\mathbf{k}}}\right|$ and $\operatorname{sgn}\left(\Delta_{\mathbf{k}}^{+}\right)=\operatorname{sgn}\left(\Delta_{\widetilde{\mathbf{k}}}^{-}\right) \neq \operatorname{sgn}\left(\Delta_{\widetilde{\mathbf{k}}}^{+}\right)=\operatorname{sgn}\left(\Delta_{\mathbf{k}}^{-}\right)$or (ii) $\mathbf{l}_{\mathbf{k}} \cdot l_{\widetilde{\mathbf{k}}}=$ $-\left|\mathbf{l}_{\mathbf{k}}\right|\left|\mathbf{l}_{\widetilde{\mathbf{k}}}\right|$ and $\operatorname{sgn}\left(\Delta_{\mathbf{k}}^{+}\right)=\operatorname{sgn}\left(\Delta_{\widetilde{\mathbf{k}}}^{+}\right) \neq \operatorname{sgn}\left(\Delta_{\mathbf{k}}^{-}\right)=\operatorname{sgn}\left(\Delta_{\widetilde{\mathbf{k}}}^{-}\right)$. Scenario (ii) includes, but is not limited to, the topological criteria 20] and 25) which desrcibe arc surface states and the Kramers-degenerate Majorana modes, respectively. In particular, the antisymmetry of the SOC vector ensures that the latter state is realized at the zone center. In contrast, states satisfying scenario (i) do not fall into either topological category: They cannot be Kramers-degenerate Majorana modes, since the requirement that $\mathbf{l}_{\mathbf{k}} \cdot \mathbf{l}_{\widetilde{\mathbf{k}}}=\left|\mathbf{l}_{\mathbf{k}}\right|\left|\mathbf{l}_{\widetilde{\mathbf{k}}}\right|$ is never satisfied at the zone center; nor can they be arc surface states protected by a $\mathbb{Z}_{2}$ number, as the condition on the gap signs implies 


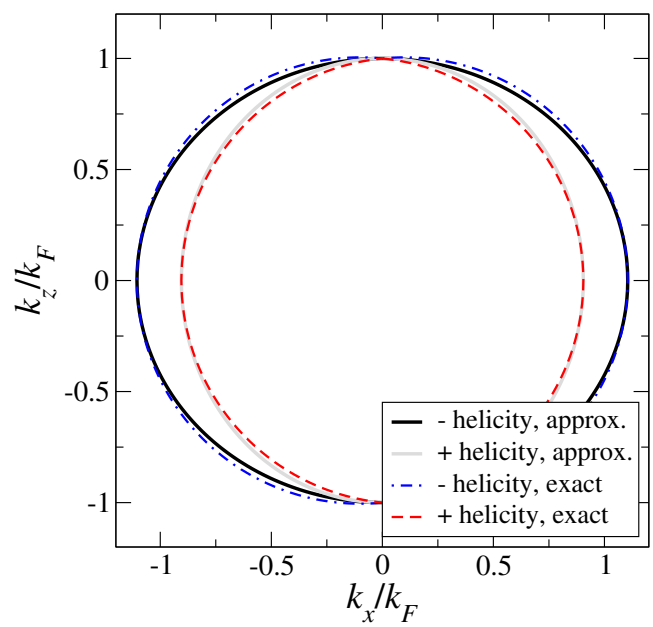

FIG. 1. (Color online) Comparison of the Fermi surfaces in the $x-z$ plane of the approximate dispersion Eq. 32 (solid lines) and the exact dispersion Eq. (31) (dashed lines) for $\lambda k_{F} / 2 \mu=0.1$.

that any plane containing $\mathbf{k}$ and $\widetilde{\mathbf{k}}$ must also contain a gap node. We note that the condition on the gaps is equivalent to $\operatorname{sgn}(f(\mathbf{k}))=-\operatorname{sgn}(f(\widetilde{\mathbf{k}}))$, which is not realized together with the condition on the polarization vector for any of the systems considered in this paper, i.e., all examples of zero-energy dispersing states presented below satisfy scenario (ii).

\section{B. Surface states for the tetragonal point group $C_{4 v}$}

In this section we analyze the bound states of the $C_{4 v}$ point group for finite spin-orbit splitting of the Fermi surface. Assuming a spherical Fermi surface in the limit of vanishing SOC we find the normal-state dispersion at finite $\lambda$,

$$
\xi_{\mathbf{k}}^{ \pm}=\frac{\mu}{k_{F}^{2}}|\mathbf{k}|^{2}-\mu \pm \lambda \sqrt{k_{x}^{2}+k_{y}^{2}},
$$

where $k_{F}=\sqrt{2 m \mu / \hbar^{2}}$. This implies a concave negativehelicity Fermi surface near $k_{z}= \pm\left|k_{F}\right|$, and so our quasiclassical method may not be well-defined across the entire surface BZ. In order to avoid this problem, we approximate the negative-helicity and positive-helicity Fermi surfaces by oblate and prolate spheroids, respectively,

$$
\xi_{\mathbf{k}}^{ \pm} \approx \frac{\mu}{\left(k_{F}^{ \pm}\right)^{2}}\left(k_{x}^{2}+k_{y}^{2}\right)+\frac{\mu}{k_{F}^{2}} k_{z}^{2}-\mu,
$$

where

$$
k_{F}^{ \pm}=k_{F}\left[\mp \frac{\lambda k_{F}}{2 \mu}+\sqrt{1+\left(\frac{\lambda k_{F}}{2 \mu}\right)^{2}}\right]
$$

gives the radius of the helical Fermi surfaces in the $k_{z}=0$ plane. Our dispersion, Eq. (32), qualitatively captures the salient features of the true dispersion $\xi_{\mathrm{k}}^{ \pm}$, Eq. 31): In the $k_{z}=0$ plane the Fermi surfaces are circles of different radii,
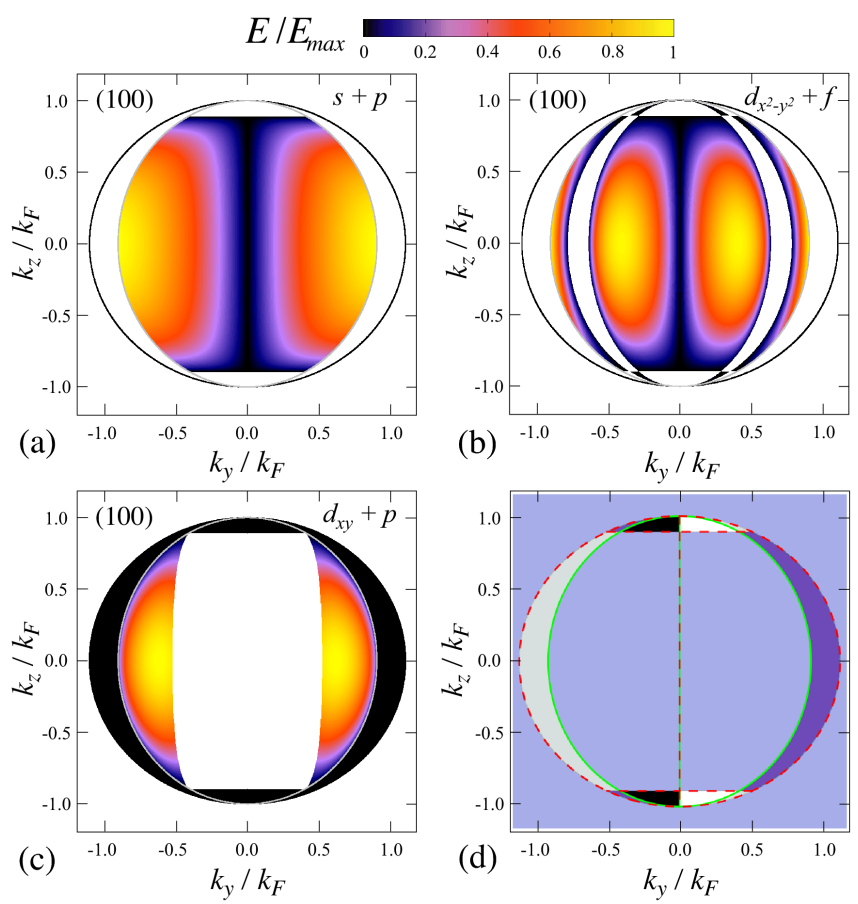

FIG. 2. (Color online) Surface bound-state spectra at the (100) face of a $C_{4 v}$ point-group NCS as a function of surface momentum $\mathbf{k}_{\|}=\left(k_{y}, k_{z}\right)$ with (a) $(s+p)$-wave, (b) $\left(d_{x^{2}-y^{2}}+f\right)$ wave, and (c) $\left(d_{x y}+p\right)$-wave pairing symmetry. Here we set $\lambda k_{F} /(2 \mu)=0.1$ and $r=0.5$. The color scale indicates the energy: black represents zero energy while yellow represents the maximum energy $E_{\max }$. The black (gray) line shows the extent of the projected negative-helicity (positive-helicity) Fermi surface. (d) Winding number $W_{(100)}$, Eq. 24, at the (100) face corresponding to the same parameters as in panel (c). Black (white) indicates $W_{(100)}=+2(-2)$, dark blue (gray) corresponds to $W_{(100)}=+1(-1)$, while light blue is $W_{(100)}=0$. The red dashed (green solid) lines represent the nodal lines on the negative-helicity (positive-helicity) Fermi surface.

and they only touch along the line $k_{x}=k_{y}=0$. As can be seen in Fig. 1, the Fermi surfaces of the approximate and exact dispersions agree very well for moderate $\lambda k_{F} / 2 \mu \leq 0.1$. To determine the bound-state spectra at given $\mathbf{k}_{\|}$, we need only find $\mathbf{k}_{\nu}, \widetilde{\mathbf{k}}_{\nu}$, and the sign of $v_{F, \perp}^{\nu}(\mathbf{k})$ at these points so that we expect that obtaining these parameters from Eq. (32) should give excellent agreement with the exact results across most of the surface BZ.

In Figs. 2(a)-(c) we present the surface bound-state spectra for the (100) face of a $C_{4 v}$ point-group NCS for several different pairing symmetries. In the case of $(s+p)$-wave pairing we observe dispersing states in the region bounded by the projected positive-helicity Fermi surface and the two nodal rings at $k_{z} \simeq \pm 0.89 k_{F}$ [Fig. 2(2)]. Since these nodal rings carry nontrivial $\mathbb{Z}_{2}$ topological charge, in agreement with the discussion in Sec. IIID there are zero-energy arc surface states ${ }^{10|20| 25 \mid 29}$ connecting the projections of the two nodal rings. Moreover, in accordance with the analysis of Secs. IIID and IV A 3, we find that this arc lies along the line $k_{y}=0$, i.e., at surface momenta that are left invariant under 

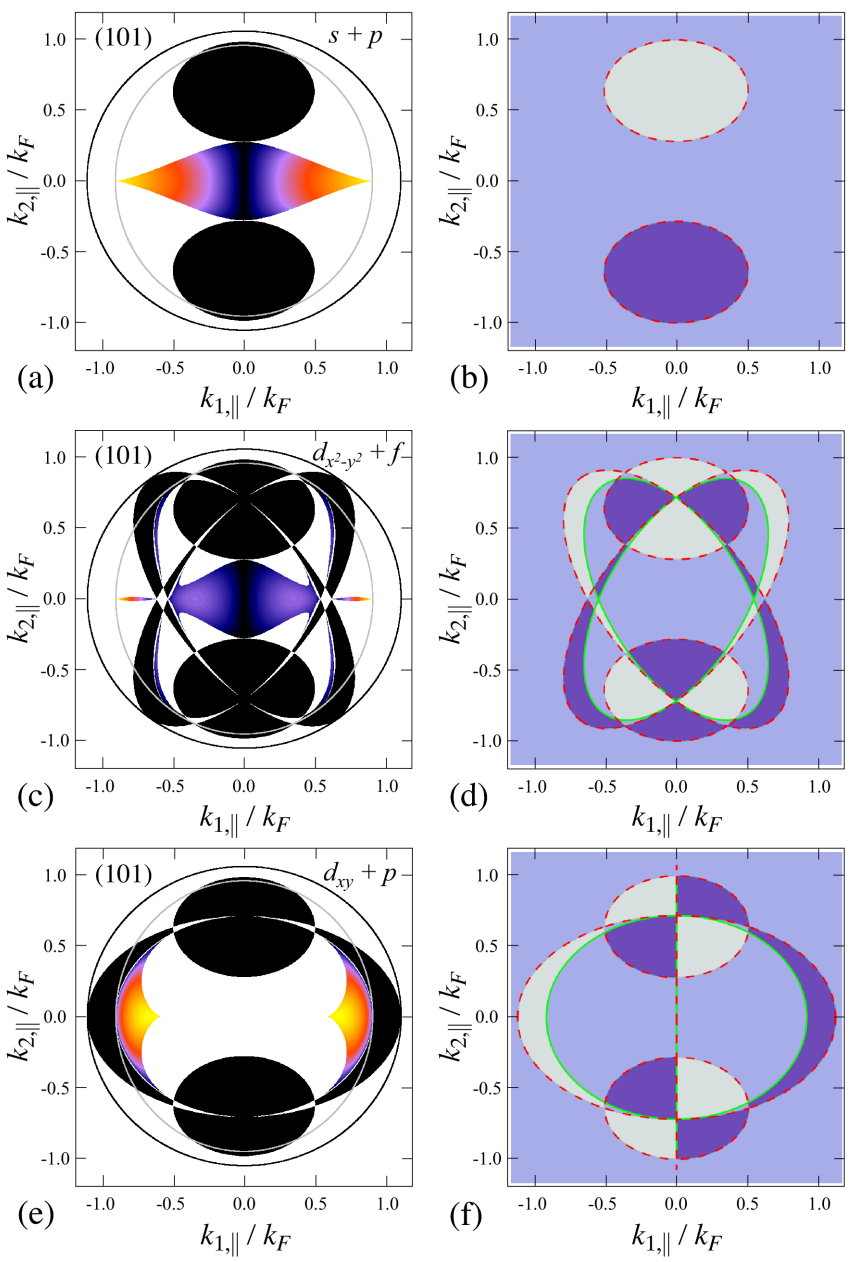

FIG. 3. (Color online) Surface bound-state spectra at the (101) face of a $C_{4 v}$ point-group NCS as a function of surface momentum $\mathbf{k}_{\|}$ with (a) $(s+p)$-wave, (c) $\left(d_{x^{2}-y^{2}}+f\right)$-wave, and (e) $\left(d_{x y}+p\right)$ wave pairing symmetry. Here we set $\lambda k_{F} /(2 \mu)=0.1$ and $r=0.5$. The color scale is the same as in Figs. 22a)-(c). The black (gray) line shows the extent of the projected negative-helicity (positive-helicity) Fermi surface. Panels (b), (d), and (f) show the winding number $W_{(101)}$ at the (101) face corresponding to the same parameters as in panels (a), (c), and (e), respectively. Dark blue (gray) indicates $W_{(101)}=+1(-1)$, while light blue is $W_{(101)}=0$. The red dashed (green solid) lines represent the nodal lines on the negative-helicity (positive-helicity) Fermi surface.

symmetry [11, and where we have $\mathbf{l}_{\mathbf{k}} \cdot \mathbf{l}_{\widetilde{\mathbf{k}}}=-\left|\mathbf{l}_{\mathbf{k}}\right|\left|\mathbf{l}_{\widetilde{\mathbf{k}}}\right|$.

The situation is qualitatively similar for the $\left(d_{x^{2}-y^{2}}+f\right)$ wave case [Fig. 2(b)], although the extra nodes due to the $d_{x^{2}-y^{2}}$ form-factor $f(\mathbf{k})$ modulate the results of the $(s+p)$ wave case. Note that these extra nodes remove the condition for the topological protection of the line of zero-energy surface states at $k_{y}=0$; the zero energy state at the zone center nevertheless remains a Kramers-degenerate Majorana mode. In contrast, Fig. 2(c) shows that we do not find any dispersing zero-energy states for the $\left(d_{x y}+p\right)$-wave pairing, but instead there are zero-energy flat bands in several regions bounded by the projected line nodes of the positive-helicity and negative-helicity gaps. The zero-energy states lying out-

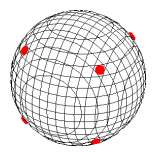

$r=\left(1+2 g_{2} / 3\right)$

topological phase topological phase topological phase transition, marginal transition, nodal transition, marginal nodal points

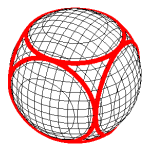

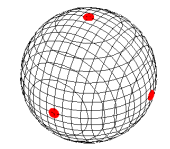

$$
r=1
$$

lines touch

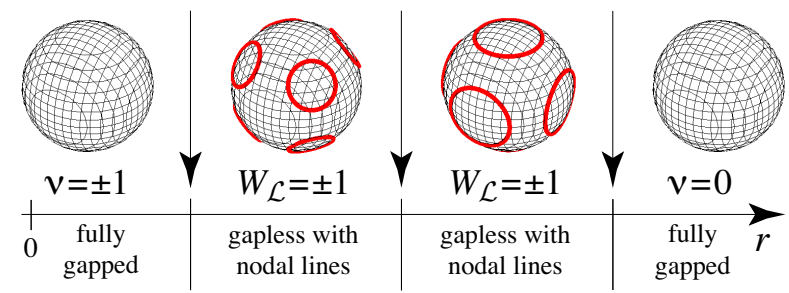

FIG. 4. (Color online) Schematic phase diagram for an NCS with cubic point group $O$ as a function of the ratio $r=\Delta_{s} / \Delta_{t}$ of the singlet and triplet gaps. Here, the second-order SOC $g_{2}$ [see Eq. [6b] is assumed to be negative.

side the projected positive-helicity Fermi surface (light gray line) are the singly degenerate "time-reversal-invariant Majorana states" found in Ref. 13, and are associated with a nontrivial winding number of $W_{(100)}= \pm 1$ as shown in Fig. 2.d). In contrast, the zero-energy surface states lying inside the projected positive-helicity Fermi surface are doubly degenerate and have winding number $W_{(100)}= \pm 2$. These states occur in the region where the gap has predominantly singlet character, and hence are due to the same mechanism as the zero-energy surface states in a pure $d_{x y}$-wave superconductor. ${ }^{4956158}$

The bound states at the (101) surface shown in Fig. 3 display a much more interesting topological character. We first consider the results for the $(s+p)$-wave case [Fig. 33(a)], which are qualitatively identical to those previously obtained in Ref. 10 for vanishing spin-orbit splitting of the Fermi surfaces. Specifically, we find that flat zero-energy bands occur within the projected nodes of the negative-helicity Fermi surface where the sign of the negative-helicity gap reverses between the forward- and backward-facing halves of the Fermi surface. These zero-energy states are associated with a finite winding number $W_{(101)}= \pm 1$; the variation of $W_{(101)}$ across the surface BZ shown in panel (b) clearly demonstrates the non-zero topological charge associated with the nodal rings of the negative-helicity gap. Like for the (100) face, the projections of these topologically charged nodal rings are connected by arc surface states. The presence of higher angular-momentum harmonics [Figs. 3(c)-(f)] results in the appearance of additional regions of zero-energy states due to the nodes of both the positive-helicity and negative-helicity gaps. All of these states correspond to a winding number of $W_{(101)}= \pm 1$, as can be seen by comparing the bound state spectra, Fig. 3.c) and (e), with the winding number calculations, Fig. 3.d) and (f), respectively. 


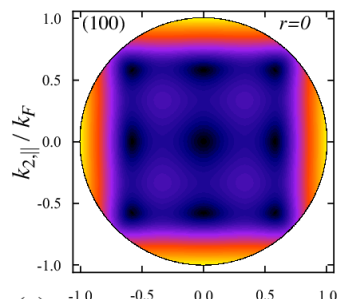

(a) $\begin{array}{lll}-1.0 & -0.5 & 0.0 \\ & & k_{1, \|} / k_{F}\end{array}$

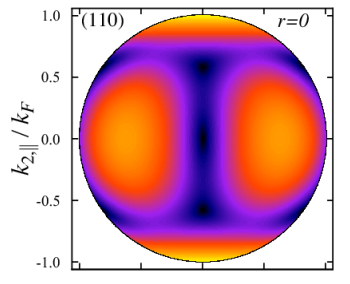

(f)
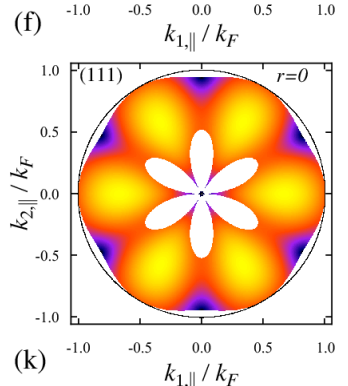

$k_{1, \|} / k_{F}$

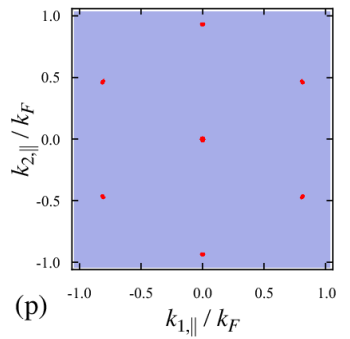

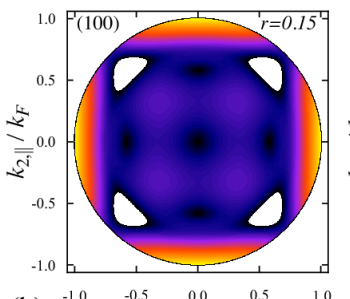

(b)
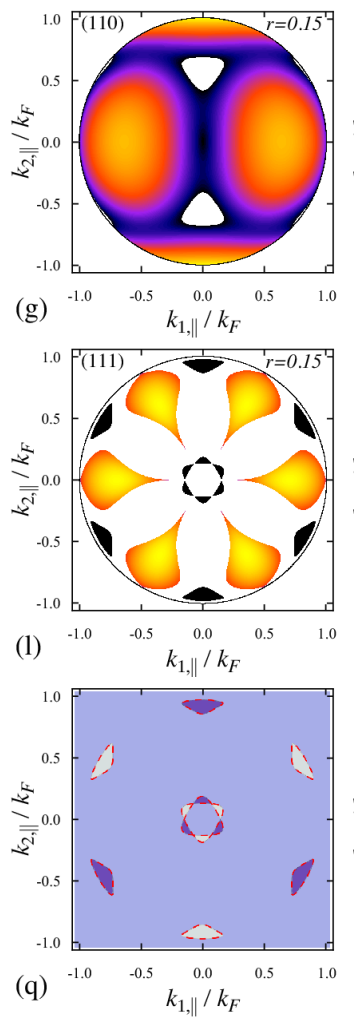

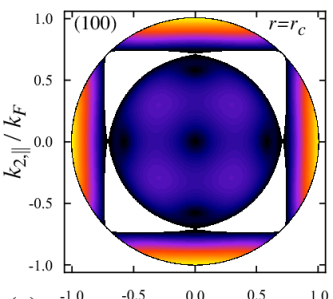

(c) ${ }^{-1.0} \quad-0.5 \quad k_{1, \|}^{0.0} / k_{F}$

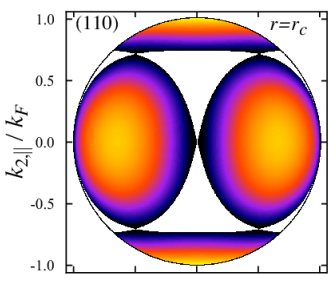

(h) $\begin{array}{llll}-1.0 & -0.5 & 0.0 \\ & & k_{1, \|} / k_{F}\end{array}$

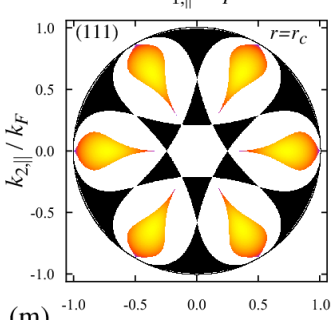

(m)

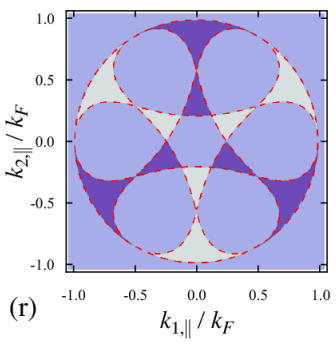

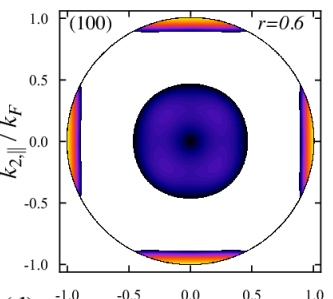

(d)

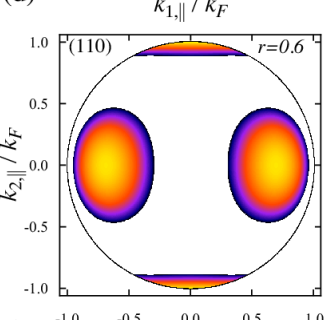

(i) ${ }^{-1.0} \quad-0.5 \quad{ }^{0.0}{ }^{0 ., \|} / k_{F}$

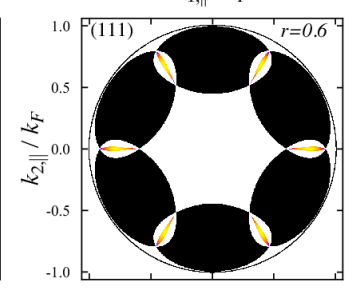

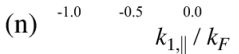

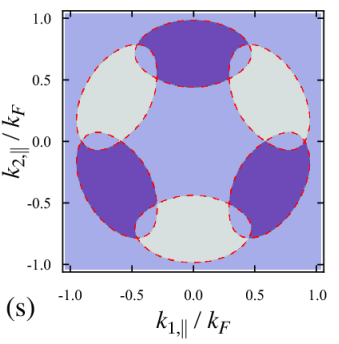

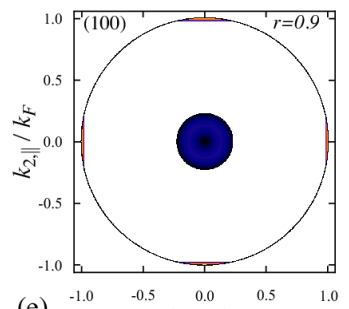

(e)
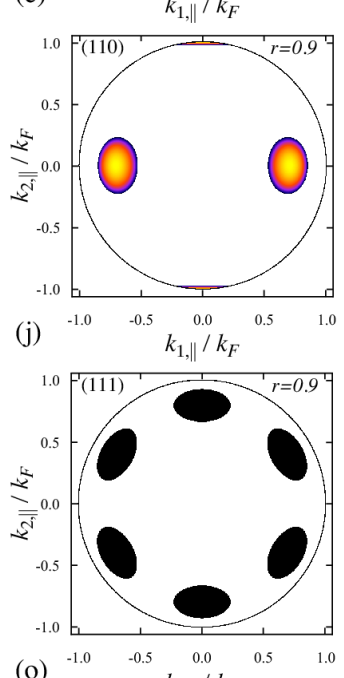

(o)

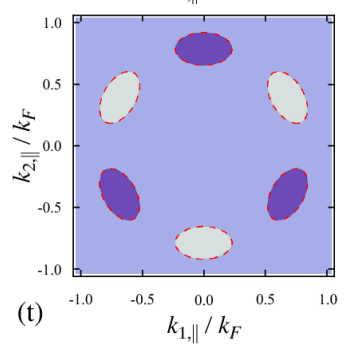

FIG. 5. (Color online) (a)-(e) Surface bound-state spectra for the (100) face of a NCS with point group $O, g_{2}=-1.5$, and $\lambda=0$, as a function of surface momentum $\mathbf{k}_{\|}$for (a) $r=0$, (b) $r=0.15$, (c) $r=r_{c}=0.25$, (d) $r=0.6$, and (e) $r=0.9$. The color scale is the same as in Figs. 22a)-(c). (f)-(j) Same as panels (a)-(e) but for the (110) face. (k)-(o) Same as panels (a)-(e) but for the (111) face. (p)-(t) Winding number $W_{(111)}$ for the (111) face corresponding to the same parameters as in panels (k)-(o). Dark blue (gray) indicates $W_{(111)}=+1(-1)$, while light blue is $W_{(111)}=0$. The red dashed lines represent the projections of nodal lines.

\section{Surface states for the cubic point group $O$}

In the cubic point group $O$ there are pronounced changes in the nodal structure of the negative-helicity gap as the singletto-triplet ratio $r=\Delta_{s} / \Delta_{t}$ is varied, which are reflected in the surface bound-state spectrum. To simplify the discussion, we assume a spherical Fermi surface, negligible spin-orbit splitting, and finite $g_{2}<00^{59}$ A schematic topological phase diagram of this NCS is presented in Fig. 4. For $r>1$ the system is fully gapped and topologically trivial. Reducing $r$, we find that at $r=1$ point nodes appear in the negative-helicity gap at $\mathbf{k}=k_{F}(1,0,0)$ and equivalent points. This is a Lifshitz transition $\frac{60}{2}$ at which the topology of the Bogoliubovde Gennes quasiparticle spectrum changes, but the symmetry of the ground state remains unaltered ${ }^{6162}$ Upon further lowering $r$, these point nodes develop into nodal rings with non-trivial topological charge. A critical value is reached at $r=r_{c}=1+g_{2} / 2$, where the nodal rings touch each other and reconnect in a different manner, i.e., there is a change in the topology of the nodal structure itself, with the rings now centered about $\mathbf{k}=\left(k_{F} / \sqrt{3}\right)(1,1,1)$ and equivalent points. Finally, at $r=1+2 g_{2} / 3$ there is another Lifshitz phase transition to a fully gapped phase with topologically non-trivial order characterized by the three-dimensional winding number $\nu= \pm 1 !^{\frac{9}{9}}$ The nodal structure at this transition point is marginal in the sense that it is topologically trivial and not protected against decay either into the fully gapped topologically non-trivial state or into the topologically stable gapless phase, i.e. point nodes do not posses any topological protection in a three-dimensional NCS. Experimental signatures of the topological phase transition will be discussed in Sec. $\mathrm{VB}$. In the following discussion of the bound states we take $g_{2}=-1.5$, which implies line nodes for $0<r<1$, point nodes for 


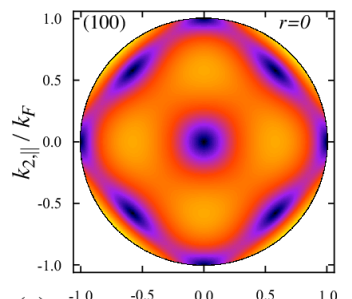

(a) ${ }^{-1.0} \quad-0.5 \quad \begin{gathered}0.0 \\ \end{gathered}$
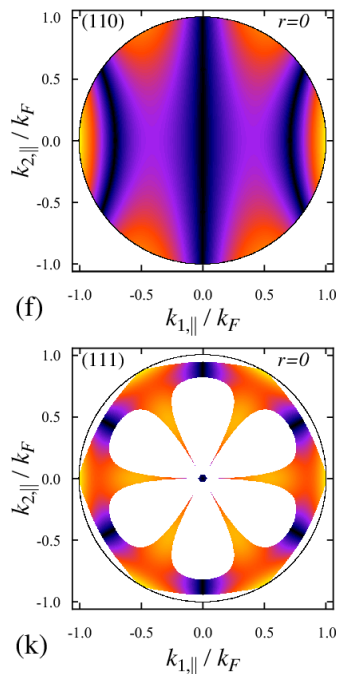

$k_{1, \|} / k_{F}$

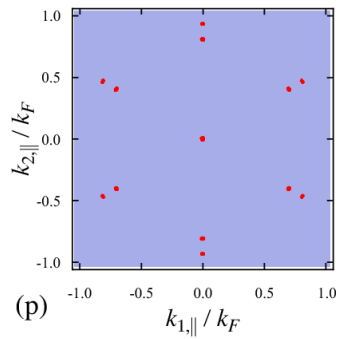

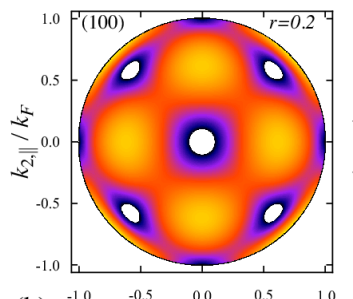

(b) ${ }^{-1.0} \quad-0.5 \quad \begin{aligned} & 0.0 \\ & \end{aligned} \quad k_{1, \|} / k_{F}$

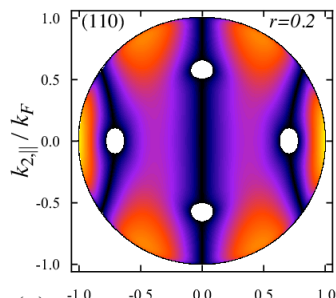

(g) ${ }^{-1.0} \quad-0.5 \quad k_{1, \|}^{0.0} / k_{F}$

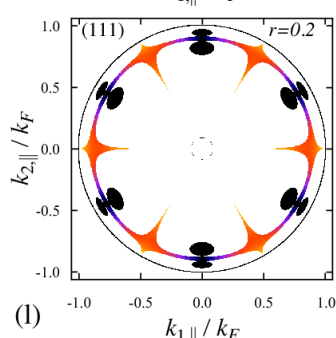

(1) $k_{1, \|} / k_{F}$

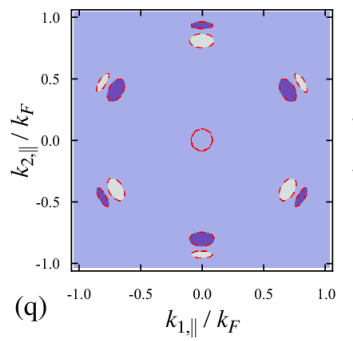

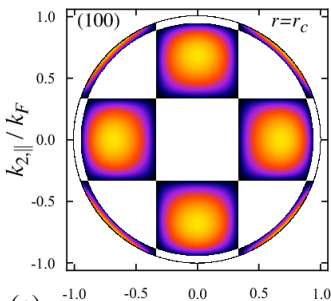

(c)

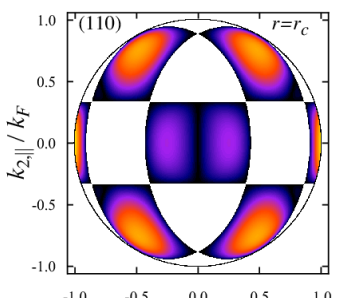

(h) ${ }^{-1.0} \quad-0.5 \quad \begin{aligned} & 0.0 \\ & \end{aligned}$

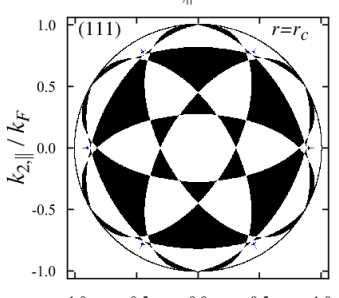

(m) $\begin{array}{llc}-1.0 & -0.5 & 0.0 \\ & & k_{1, \|} / k_{F}\end{array}$

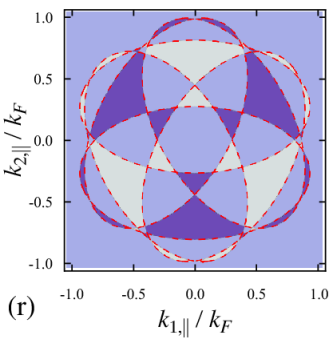

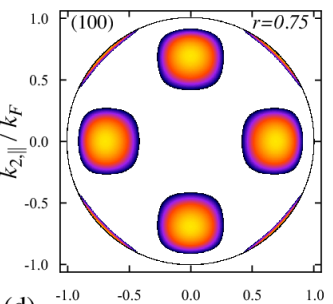

(d) ${ }^{-1.0} \quad-0.5 \quad \begin{aligned} & 0.0 \\ & \end{aligned}$

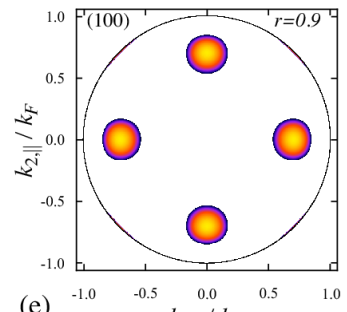

(e)

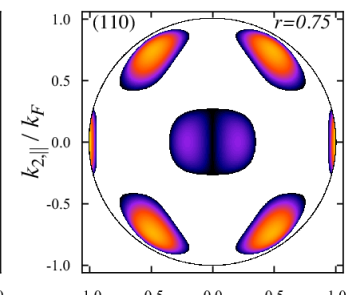

(i) ${ }^{-1.0} \quad-0.5{ }^{0.0}{ }_{1, \|} / k_{F}$

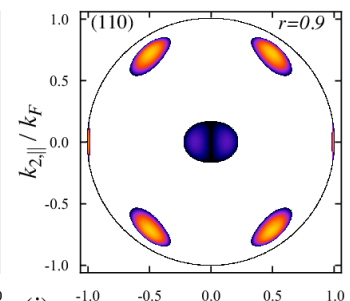

(j)
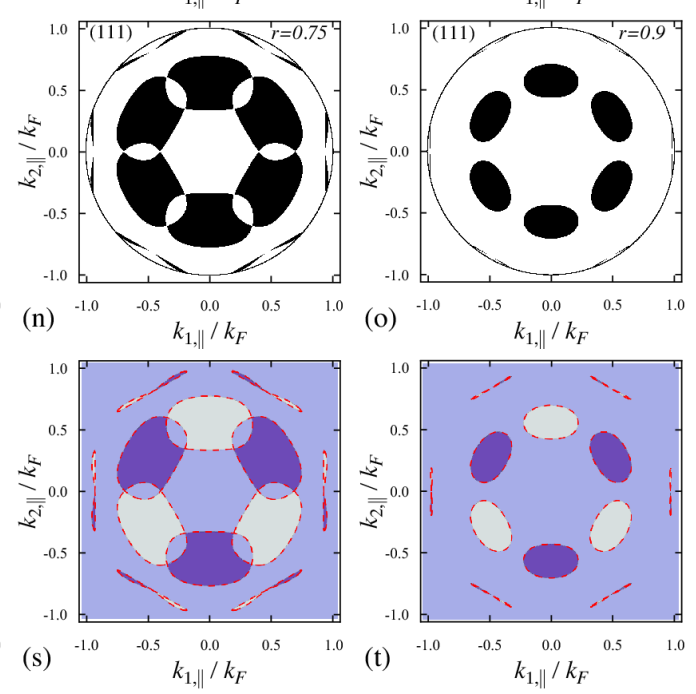

FIG. 6. (Color online) (a)-(e) Surface bound-state spectra for the (100) face of a NCS with point group $T_{d}$ and $\lambda=0$ as a function of the surface momentum $\mathbf{k}_{\|}$for (a) $r=0$, (b) $r=0.2$, (c) $r=r_{c}=4 \sqrt{2} / 9$, (d) $r=0.75$, and (e) $r=0.9$. The color scale is the same as in Figs. 2(a)-(c). (f)-(j) Same as panels (a)-(e) but for the (110) face. (k)-(o) Same as panels (a)-(e) but for the (111) face. (p)-(t) Winding number $W_{(111)}$ for the (111) face corresponding to the same parameters as in panels (k)-(o). Dark blue (gray) indicates $W_{(111)}=+1(-1)$, while light blue is $W_{(111)}=0$. The red dashed lines represent the projections of nodal lines.

$r=0,1$, and a fully gapped state for $r>1$. Surface bound states only occur for $r<1$.

We first consider the bound states at the (100) face [Figs. 5(a)-(e)], which were partially examined in Ref. 27 for $r>r_{c}$. Starting at $r=0$ we find bound states for all $\left|\mathbf{k}_{\|}\right|<k_{F}$ except at the projected point nodes. Of particular note are the dispersing zero energy states at $\left(k_{1, \|}, k_{2, \|}\right)=$ $(0,0),\left(k_{F} / \sqrt{3}, 0\right)$ and equivalent points, where the condition $l_{\mathbf{k}}=-l_{\widetilde{\mathbf{k}}}$ is satisfied. From Sec. IIII however, we deduce that only the zero-energy state at the zone center is a topologically protected Majorana fermion. The other four zero-energy states, in contrast, only form for $g_{2}<-1$ and hence are not topologically stable. The apparent zeros close to $\left(k_{F} / \sqrt{3}, k_{F} / \sqrt{3}\right)$ and symmetry-related points in (a) are the projections of the point nodes. Upon increasing $r$, the nodal rings on the negative-helicity Fermi surface lead to regions within the projected Fermi surface where bound states do not occur, i.e., the white space in Fig. 5. (b). Note that we have $W_{(100)}=0$ everywhere since the integration path $\mathcal{L}$ passes through none or two nodal rings, the contributions of which cancel. The reconnection of the nodal rings at $r=r_{c}$ splits the bound states into disconnected regions at the zone center and near the edges of the projected Fermi surface. Note that the Majorana fermion mode at the zone center survives up to $r=1$. As shown in Figs. 5 ff)-(j), the situation for the (110) face is essentially similar, although here the Majorana fermion state at the zone center only survives up to the topological Lifshitz transition at $r=r_{c}$.

The bound states at the (111) face are qualitatively different due to the presence of topologically protected zeroenergy bands. We show the bound state spectra and the associated maps of the winding number $W_{(111)}$ in Figs. $5(\mathrm{k})$ (o) and Figs. 5(p)-(t), respectively. Unlike for the other two 

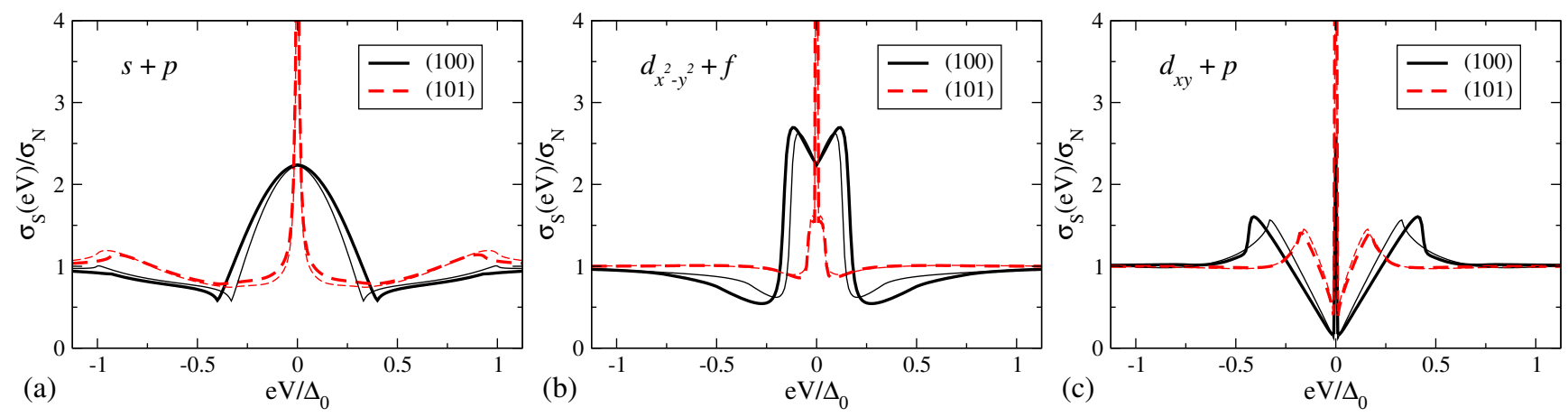

FIG. 7. (Color online) Tunneling conductance spectra for the (100) and (101) interfaces of a $C_{4 v}$ NCS with $r=0.5, Z=3$, and (a) $(s+p)$ wave gap symmetry $[f(\mathbf{k})=1]$, (b) $\left(d_{x^{2}-y^{2}}+f\right)$-wave gap symmetry $\left[f(\mathbf{k})=\left(k_{x}^{2}-k_{y}^{2}\right) / k_{F}^{2}\right]$, and (c) $\left(d_{x y}+p\right)$-wave gap symmetry $\left[f(\mathbf{k})=2 k_{x} k_{y} / k_{F}^{2}\right]$. In all panels the thick lines show the results for finite spin-orbit splitting $\lambda k_{F} / 2 \mu=0.1$, while the thin lines show the results for degenerate helical Fermi surfaces.

surfaces, we find that at $r=0$ the energy of the dispersing states reaches the gap so that we have regions within the projected Fermi surface where bound states do not occur. For finite but small $r$, the dispersing bound states break up into six lobes, with zero-energy flat surface bands within the projected nodal lines close to the center and edges. Like for the $C_{4 v}$ point group, the zero-energy states are associated with a winding number $W_{(111)}= \pm 1$. Increasing $r$ we see that the zero-energy surface states grow in extent while the dispersing states shrink, until at $r=r_{c}$ the nodes in the gap touch and the zero-energy states surround the dispersing states. The dispersing states vanish at $r$ closer to 1 when the projected nodes no longer overlap, and we hence find only zero-energy states in Fig. 5(o).

\section{Surface states for the tetrahedral point group $T_{d}$}

The tetrahedral point group $T_{d}$ also shows topological phase transitions in the nodal structure upon varying $r$, which we again discuss for a spherical Fermi surface and vanishing spin-orbit splitting. At $r>1$ the system is fully gapped and topologically trivial. Upon reducing the singlet-to-triplet ratio to $r=1$, a Lifshitz transition occurs with the appearance of twelve point nodes in the negative-helicity gap at $\mathbf{k}=\left(k_{F} / \sqrt{2}\right)(1,1,0)$ and equivalent points; further reducing $r$ we find topologically charged nodal rings centered about these points. These rings grow as $r$ is lowered, eventually touching at $r=r_{c}=4 \sqrt{2} / 9$. For $r<r_{c}$ the nodal rings reconnect in a different way so that they are now centered about the fourteen zeros of $\mathbf{l}_{\mathbf{k}}$ at $\mathbf{k}=k_{F}(1,0,0),\left(k_{F} / \sqrt{3}\right)(1,1,1)$ and equivalent points. In the limit of a purely triplet gap we find point nodes at these locations.

The bound states at the (100) surface are shown in Figs. 6(a)-(e). Unlike for the cubic point group $O$, there are no zero-energy dispersing states for this surface; the apparent zero-energy states at $r=0$ in Fig. 6a) are in fact nodes. For $r>0$ these points become lines, clearly visible as the boundaries of the white space. As $r$ is increased past $r_{c}$ the dispersing bound states separate into disconnected regions of the BZ, which vanish at $r=1$. We now turn to the bound states for the (110) surface, which are presented in Figs. 6(f)(j). At $r<r_{c}$ we find lines of dispersing zero-energy states which connect the projected point nodes (for $r=0$ ) and nodal rings (for $r>0$ ) of the negative-helicity gap. These arc states are not topologically protected by the mechanism discussed in Sec. IIID however, since for this point-group symmetry the condition Eq. (11b) only holds for planes which intersect line nodes of the gap. We nevertheless note that the zero-energy state at the surface BZ center is a topologically protected Majorana fermion which is present for all $r<1$.

Like for the cubic point group $O$, topologically protected zero-energy bound states are found at the (111) surface for $0<r<1$. The bound state spectra are plotted in Figs. 6(k)(o), with the variation of the winding number $W_{(111)}$ shown in Figs. 6(p)-(t). The main difference to the case of the point group $O$ is the much more limited extent of the dispersing bound states, which are almost entirely absent for $r>r_{c}$. At small $r$ we also observe arc surface states connecting the topologically charged nodal rings near the edge of the projected Fermi surface, but again these states are not topologically protected by the mechanism discussed in Sec. IIID

\section{TUNNELING CONDUCTANCE AND TOPOLOGICAL PHASE TRANSITIONS}

In this section we discuss the calculation of the tunnelingconductance spectra for a normal-metal-NCS junction. Such tunneling experiments are an important test for the pairing symmetry of unconventional superconductors ${ }^{[56}$ In particular, they can confirm the existence of the zero-energy surface flat bands, which are evidenced by a sharp peak in the low-temperature conductance at zero bias! $\frac{10113}{10}$ We also discuss possible signatures of the topological phase transitions. 


\section{A. Tunneling conductance}

At zero temperature the tunneling conductance $\sigma_{S}(\mathrm{eV})$ for tunneling from a normal metal without SOC into an NCS under bias voltage $V$ is given by the generalized BlonderTinkham-Klapwijk formula 2012539963

$$
\sigma_{S}(e V)=\sum_{\mathbf{k}_{\|}} \sum_{\sigma, \sigma^{\prime}}\left\{1+\left|a_{\sigma, \sigma^{\prime}}\left(\mathbf{k}_{\|}\right)\right|^{2}-\left|b_{\sigma, \sigma^{\prime}}\left(\mathbf{k}_{\|}\right)\right|^{2}\right\},
$$

where $a_{\sigma, \sigma^{\prime}}\left(\mathbf{k}_{\|}\right)$and $b_{\sigma, \sigma^{\prime}}\left(\mathbf{k}_{\|}\right)$are the spin-resolved Andreev and normal reflection coefficients for electron injection into the NCS, respectively. These coefficients appear in the wavefunction ansatz describing the electron-injection process,

$$
\Psi_{\sigma}\left(\mathbf{k}_{\|}, \mathbf{r}\right)=\psi_{\sigma}^{<}\left(\mathbf{k}_{\|}, \mathbf{r}\right) \Theta\left(-r_{\perp}\right)+\psi_{\sigma}^{>}\left(\mathbf{k}_{\|}, \mathbf{r}\right) \Theta\left(r_{\perp}\right) .
$$

In the normal metal the wavefunction is written as

$$
\begin{aligned}
\psi_{\sigma}^{<}\left(\mathbf{k}_{\|}, \mathbf{r}\right)= & \psi_{e, \sigma} e^{i \mathbf{k} \cdot \mathbf{r}}+\sum_{\sigma^{\prime}}\left[a_{\sigma, \sigma^{\prime}}\left(\mathbf{k}_{\|}\right) \psi_{h, \sigma^{\prime}} e^{i \mathbf{k} \cdot \mathbf{r}}\right. \\
& \left.+b_{\sigma, \sigma^{\prime}}\left(\mathbf{k}_{\|}\right) \psi_{e, \sigma^{\prime}} e^{i \widetilde{\mathbf{k}} \cdot \mathbf{r}}\right]
\end{aligned}
$$

with the electron and hole spinors $\psi_{e, \sigma}=\frac{1}{2}(1+\sigma, 1-\sigma, 0,0)^{T}$ and $\psi_{h, \sigma}=\frac{1}{2}(0,0,1+\sigma, 1-\sigma)^{T}$, respectively. In the case that there are propagating solutions in both helicity sectors, the wavefunction in the NCS is written as

$$
\psi_{\sigma}^{>}\left(\mathbf{k}_{\|}, \mathbf{r}\right)=\sum_{n= \pm}\left[c_{\sigma, n} \psi_{n}\left(\mathbf{k}_{n}\right) e^{i \mathbf{k}_{n} \cdot \mathbf{r}}+d_{\sigma, n} \psi_{n}\left(\widetilde{\mathbf{k}}_{n}\right) e^{i \widetilde{\mathbf{k}}_{n} \cdot \mathbf{r}}\right],
$$

where the spinors are defined as in Sec. IVA1, if there are propagating solutions only in the negative-helicity sector, the positive-helicity components of Eq. (37) are replaced by Eq. 28a. To simplify the discussion we assume that the normal metal has a spherical Fermi surface with the same chemical potential and effective mass as the NCS. We restrict ourselves to the limit where the bias energy is negligible compared to the Fermi energy so that the energy dependence of the wavevectors can be ignored.

The reflection coefficients are determined by application of the interface boundary conditions. The first condition requires continuity of the wavefunction across the interface, i.e.,

$$
\left.\psi_{\sigma}^{<}\left(\mathbf{k}_{\|}, \mathbf{r}\right)\right|_{r_{\perp}=0^{-}}=\left.\psi_{\sigma}^{>}\left(\mathbf{k}_{\|}, \mathbf{r}\right)\right|_{r_{\perp}=0^{+}} .
$$

The second condition enforces conservation of probability across the barrier, $64 \sqrt[66]{6 h i c h}$ is modeled as a $\delta$-function of height $U$. We have

$$
\left.\check{v}_{S} \psi_{\sigma}^{>}\left(\mathbf{k}_{\|}, \mathbf{r}\right)\right|_{r_{\perp}=0^{+}}=\left.\left(\partial_{r_{\perp}}+2 Z k_{F}\right) \psi_{\sigma}^{<}\left(\mathbf{k}_{\|}, \mathbf{r}\right)\right|_{r_{\perp}=0^{-}},
$$

where $Z=m U / \hbar^{2} k_{F}$ is a dimensionless parameter characterizing the transparency of the interface, and $\check{v}_{S}$ is proportional to the $r_{\perp}$ component of the velocity operator in the normal state of the NCS. For a $C_{4 v}$ point-group NCS orientated such that the $b$ axis is parallel to the interface and the $c$ axis makes an angle $\alpha$ to the interface, we write

$$
\check{v}_{S}=\hat{\sigma}_{0} \otimes \hat{\sigma}_{0} \partial_{r_{\perp}}+i \hat{\sigma}_{0} \otimes \hat{\sigma}_{2} \frac{m \lambda}{\hbar^{2}} \cos \alpha .
$$

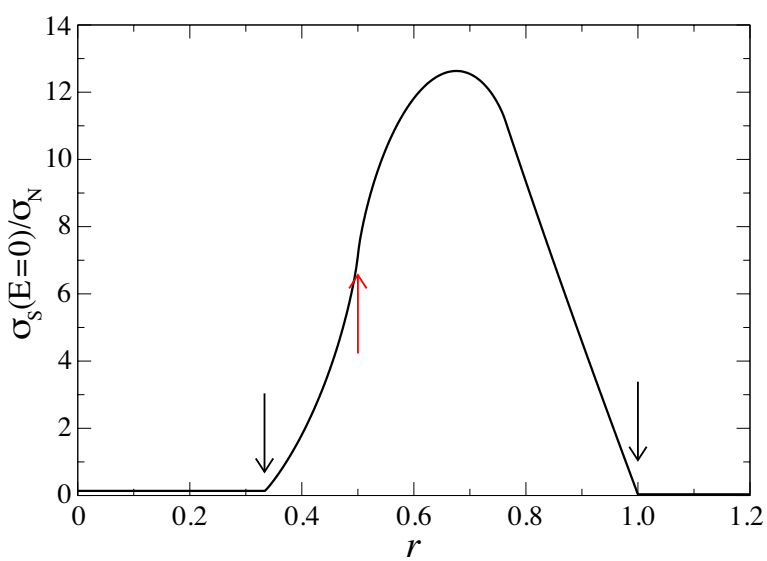

FIG. 8. (Color online) Variation of the zero-bias conductance-peak height at the (111) face as a function of singlet-to-triplet ratio $r$ for the point group $O$. The black, downward-pointing arrows indicate topological phase transitions between fully gapped and nodal phases. The red, upward-pointing arrow marks an inflection point in $\sigma_{S}(\mathrm{eV})$, where the character of the nodes on $\Delta_{\mathrm{k}}^{-}$changes qualitatively. In this figure we set $g_{2}=-1, Z=3$, and $T=0 \mathrm{~K}$.

In Fig.7 7 we present conductance spectra for tunneling into a $C_{4 v}$ point-group NCS through (100) and (101) interfaces for both vanishing and finite SOC $\lambda$. We find the tunnelingconductance results to be quite robust against the finite spinorbit splitting of the Fermi surfaces. Several features of these spectra are noteworthy. For the (100) surface we observe a broad hump-like feature in the tunneling conductance for the $(s+p)$-wave and $\left(d_{x^{2}-y^{2}}+f\right)$-wave pairing states, which is a signature of the arc surface states. In the $\left(d_{x y}+p\right)$-wave case, in contrast, we find a zero-bias conductance peak wellseparated from the bulk density of states. In the limit of degenerate helical Fermi surfaces this is due to the doubly degenerate zero-energy states for $\mathbf{k}_{\|}$close to $\pm k_{F} \mathbf{e}_{z}$ [see Fig. 2(d)], while for non-zero SOC the singly degenerate zero-energy states near $\mathbf{k}_{\|}= \pm k_{F} \mathbf{e}_{y}$ also contribute. For the (101) surface all pairing symmetries show a zero-bias conductance peak, which is a key experimental signature of the topologically protected zero-energy states $!^{[10 \mid 13}$ For the cases of $(s+p)$-wave and $\left(d_{x^{2}-y^{2}}+f\right)$-wave pairing, however, we note that this is superimposed on a much diminished hump-like feature. This signals the continued existence of arc surface states in these systems, in agreement with Fig. 3 .

\section{B. Evidence for topological phase transitions}

In Sec. IV C we have demonstrated the existence of topological phase transitions as a function of the singlet-triplet ratio $r$ for an NCS with point group $O$ and SOC vector $\mathbf{l}_{\mathbf{k}}$ given by Eq. (6b) with $g_{2} \neq 0$. This discussion is physically relevant for $\mathrm{Li}_{2} \mathrm{Pd}_{x} \mathrm{Pt}_{3-x} \mathrm{~B}$, for which the SOC strength can be tuned by substituting Pd for Pt ${ }^{67}$ The magnitude of the SOC interaction in these compounds seems to be directly related to $r, \frac{36}{36}$ which suggests that it might be possible to observe topo- 
logical phase transitions between a fully gapped and a gapless phase, or between two gapless phases, in $\mathrm{Li}_{2} \mathrm{Pd}_{x} \mathrm{Pt}_{3-x} \mathrm{~B}$ as a function of Pt concentration.

The most direct way to detect the topological phase transitions in this system requires measurements sensitive to the low-energy bulk density of states $\rho(\omega)$. At the $r=1$ and $r=\left(1+2 g_{2} / 3\right)$ Lifshitz transitions, this quantity changes from $\rho(\omega)=0$, characteristic of a full gap, to the linear dependence $\rho(\omega) \propto \omega$ associated with line nodes. Signatures of the topological phase transition can also be seen in the conductance spectra, in particular the zero-bias conductance peak at the (111) surface. Indeed, as shown in Fig. 8, the zero-bias conductance shows abrupt changes at the boundaries of the nodal region. Furthermore, at the critical $r_{c}$ where the topological structure of the nodes changes, there is a kink anomaly which is marked by the red, upward-pointing arrow in Fig. 8 . Tunneling experiments could therefore in principle be used to evidence a topological phase transition in $\mathrm{Li}_{2} \mathrm{Pd}_{x} \mathrm{Pt}_{3-x} \mathrm{~B}$.

\section{CONCLUSIONS AND OUTLOOK}

We have performed a detailed analysis of the topological properties of nodal NCSs in three dimensions. Using topological arguments, we have derived general criteria for the existence of Andreev bound states at the surface of nodal NCSs. Three different types of topologically protected surface states have been identified, namely Kramers-degenerate Majorana modes, arc surface states, and surface flat bands, whose stability is guaranteed by the conservation of the one- and twodimensional $\mathbb{Z}_{2}$ invariants [Eq. (22) and [18], and the winding number (14), respectively.

We have independently derived the general criteria for zeroenergy surface states using the quasiclassical scattering theory. Furthermore, we have applied this technique to study a number of physically relevant manifestations of NCSs. For a $C_{4 v}$ point-group symmetry, we have calculated the surface bound-state spectra and the tunneling conductance for $(s+p)$ wave, $\left(d_{x^{2}-y^{2}}+f\right)$-wave, and $\left(d_{x y}+p\right)$-wave pairing and finite spin-orbit splitting. We have shown that the surface bound states are in perfect agreement with the variation of the topological winding number across the surface BZ. We have shown how the surface flat bands manifest themselves as a zero-bias conductance peak, while the arc surface states lead to a broad, hump-like feature centered around zero bias in the conductance spectra. Both features exhibit a pronounced dependence on surface orientation, which provides characteristic fingerprints of the topological properties of the system. We have also examined the bound-state spectra in NCSs with $O$ and $T_{d}$ point-group symmetry in the limit of weak spin-orbit splitting and discussed the occurrence of topological Lifshitz transitions in these spectra.

On symmetry grounds the zero-energy surface flat bands are expected to appear in any unconventional nodal superconductor preserving TRS. In particular, this should be the case for the class-CI topological superconductors. ${ }^{68}$ Furthermore, flat bands are also expected to occur at the surface of nodal topological superconductors in symmetry class AIII $[90$ (i.e., topological superconductors with TRS that are invariant under rotations about one fixed axis in spin space). Besides the tunneling conductance, the surface flat bands and arc surface states also profoundly affect other surface and interface properties of NCSs, such as Josephson tunneling, 71 the nonlinear Meissner effect, and surface thermal transport. The investigation of these interesting boundary properties are left for future work.

\section{ACKNOWLEDGMENTS}

The authors thank L. Klam, S. Ryu, M. Sigrist, and G. Khaliullin for useful discussions. *.schnyder@fkf.mpg.de

$\dagger$ brydon@ theory.phy.tu-dresden.de

$\ddagger$ carsten.timm@tu-dresden.de

1 M. Hasan and C. Kane, Rev. Mod. Phys. 82, 3045 (2010).

2 X.-L. Qi and S.-C. Zhang, Rev. Mod. Phys. 83, 1057 (2011).

${ }^{3}$ M. Z. Hasan and J. E. Moore, Ann. Rev. Cond. Matt. Phys. 2, 55 (2011).

4 M. Sigrist, AIP Conf. Proc. 1162, 55 (2009).

5 V. P. Mineev, M. Sigrist, e-print arXiv:0904.2962

${ }^{6}$ M. Sato, Phys. Rev. B 73, 214502 (2006).

7 B. Beri, Phys. Rev. B 81, 134515 (2010).

${ }^{8}$ M. Sato and S. Fujimoto, Phys. Rev. B 79, 094504 (2009).

9 A. P. Schnyder and S. Ryu, Phys. Rev. B 84, 060504(R) (2011).

${ }^{10}$ P. M. R. Brydon, A. P. Schnyder, and C. Timm, Phys. Rev. B 84, 020501(R) (2011).

11 M. Sato, Y. Tanaka, K. Yada, and T. Yokoyama, Phys. Rev. B 83, 224511 (2011).

${ }^{12}$ K. Yada, M. Sato, Y. Tanaka, and T. Yokoyama, Phys. Rev. B 83, 064505 (2011).
13 Y. Tanaka, Y. Mizuno, T. Yokoyama, K. Yada, and M. Sato, Phys. Rev. Lett. 105, 097002 (2010).

${ }^{14}$ M. Sato and S. Fujimoto, Phys. Rev. Lett. 105, 217001 (2010).

15 A. P. Schnyder, S. Ryu, A. Furusaki, and A. W. W. Ludwig, Phys. Rev. B 78, 195125 (2008).

16 A. P. Schnyder, S. Ryu, A. Furusaki, and A. W. W. Ludwig, AIP Conf. Proc. 1134, 10 (2009).

17 S. Ryu, A. P. Schnyder, A. Furusaki, and A. W. W. Ludwig, New J. Phys. 12065010 (2010).

18 R. Roy, e-print arXiv:0803.2868

19 X.-L. Qi, T. L. Hughes, S. Raghu, and S.-C. Zhang, Phys. Rev. Lett. 102187001 (2009).

${ }^{20}$ M. Eschrig, C. Iniotakis, and Y. Tanaka, e-print arXiv:1001.2486

${ }^{21}$ C. Kane and E. Mele, Phys. Rev. Lett. 95, 146802 (2005).

${ }^{22}$ M. Sato, Phys. Rev. B 79, 214526 (2009).

23 X.-L. Qi, T. L. Hughes, and S.-C. Zhang, Phys. Rev. B 81, 134508 (2010).

${ }^{24}$ Here and in the following the term "nodal line" refers to the vanishing of the superconducting gap along one-dimensional lines on the Fermi surface. 
${ }^{25}$ C. Iniotakis, N. Hayashi, Y. Sawa, T. Yokoyama, U. May, Y. Tanaka, and M. Sigrist, Phys. Rev. B 76, 012501 (2007).

26 A. B. Vorontsov, I. Vekhter, and M. Eschrig, Phys. Rev. Lett. 101, 127003 (2008); Physica B 403, 1095 (2008).

27 A. B. Vorontsov, I. Vekhter, and M. Eschrig, J. Phys. Soc. Japan Suppl. A 77, 165 (2008).

${ }^{28}$ C.-K. Lu and S.-K. Yip, Phys. Rev. B 82, 104501 (2010).

29 Y. Tanaka, T. Yokoyama, A. V. Balatsky, and N. Nagaosa, Phys. Rev. B 79, 060505(R) (2009).

${ }^{30}$ E. Bauer, G. Hilscher, H. Michor, Ch. Paul, E. W. Scheidt, A. Gribanov, Y. Seropegin, H. Noël, M. Sigrist, and P. Rogl, Phys. Rev. Lett. 92, 027003 (2004).

${ }^{31}$ N. Kimura, K. Ito, K. Saitoh, Y. Umeda, H. Aoki, and T. Terashima, Phys. Rev. Lett. 95, 247004 (2005).

${ }^{32}$ I. Sugitani, Y. Okuda, H. Shishido, T. Yamada, A. Thamizhavel, E. Yamamoto, T. D. Matsuda, Y. Haga, T. Takeuchi, R. Settai, and Y. Onuki, J. Phys. Soc. Jpn. 75, 043703 (2006).

${ }^{33}$ G. Amano, S. Akutagawa, T. Muranaka, Y. Zenitani, and J. Akimitsu, J. Phys. Soc. Jpn. 73, 530 (2004).

${ }^{34}$ K. Togano, P. Badica, Y. Nakamori, S. Orimo, H. Takeya, and K. Hirata, Phys. Rev. Lett. 93, 247004 (2004).

35 P. Badica, T. Kondo, and K. Togano, J. Phys. Soc. Jpn. 74, 1014 (2005).

${ }^{36}$ H. Q. Yuan, D. F. Agterberg, N. Hayashi, P. Badica, D. Vandervelde, K. Togano, M. Sigrist, and M. B. Salamon, Phys. Rev. Lett. 97, 017006 (2006).

${ }^{37}$ E. Bauer, G. Rogl, X.Q. Chen, R. T. Khan, H. Michor, G. Hilscher, E. Royanian, K. Kumagai, D. Z. Li, Y. Y. Li, R. Podloucky, and P. Rogl, Phys. Rev. B 82, 064511 (2010).

38 A. B. Karki, Y. M. Xiong, I. Vekhter, D. Browne, P. W. Adams, D. P. Young, K. R. Thomas, J. Y. Chan, H. Kim, and R. Prozorov, Phys. Rev. B 82, 064512 (2010).

39 T. Yokoyama, Y. Tanaka, and J. Inoue, Phys. Rev. B 72, 220504(R) (2005).

${ }^{40}$ K. Børkje and A. Sudbø, Phys. Rev. B 74, 054506 (2006).

41 J. Linder and A. Sudbø, Phys. Rev. B 76, 054511 (2007).

${ }^{4}$ P. A. Frigeri, D. F. Agterberg, A. Koga, and M. Sigrist, Phys. Rev. Lett. 92, 097001 (2004).

${ }^{43}$ K. V. Samokhin, Annals of Physics 324, 2385 (2009).

${ }^{44}$ M. R. Zirnbauer, J. Math. Phys. 37, 4986 (1996).

45 A. Altland and M. R. Zirnbauer, Phys. Rev. B 55, 1142 (1997).

46 The first entry in the tensor product $\sigma_{i} \otimes \sigma_{j}$ corresponds to Nambu space, whereas the second one corresponds to spin space.

${ }^{47}$ M. Nakahara, Geometry, Topology, and Physics, 2nd ed. (Institute of Physics, Bristol, 2003).
${ }^{48}$ For instance, $q(\mathbf{k})$ of a $C_{4 v}$ point-group NCS with $(s+p)$-wave paring can be regularized on a cubic lattice by letting $\varepsilon_{\mathbf{k}} \rightarrow$ $t_{1}\left(\cos k_{x}+\cos k_{y}+\cos k_{z}\right)-\mu$ and $\mathbf{l}_{\mathbf{k}} \rightarrow\left(\sin k_{y},-\sin k_{x}, 0\right)^{\mathrm{T}}$.

49 S. Ryu and Y. Hatsugai, Phys. Rev. Lett. 89, 077002 (2002).

${ }^{50}$ In deriving Eqs. (24) and (25), we have assumed that for any given $\mathbf{k}_{\|}, \xi_{\mathbf{k}_{ \pm}}^{ \pm}=0$ and $\xi_{\tilde{\mathbf{k}}_{+}}^{ \pm}=0$ each have a maximum of two solutions. This is justified for a single-band NCS with two convex Fermi surfaces.

51 G. E. Volovik, Pis'ma ZhETF 93, 69 (2011) [JETP Lett. 93, 66 (2011)].

${ }^{52}$ X. Wan, A. Turner, A. Vishwanath, and S. Y. Savrasov, Phys. Rev. B 83, 205101 (2011).

53 A. A. Burkov and L. Balents, Phys. Rev. Lett. 107, 127205 (2011).

${ }^{54}$ M. Wimmer, A. R. Akhmerov, F. Guinea, Phys. Rev. B 82, 045409 (2010).

55 M. Matsumoto and H. Shiba, J. Phys. Soc. Jpn. 64, 1703 (1995).

56 S. Kashiwaya and Y. Tanaka, Rep. Prog. Phys. 63, 1641 (2000).

57 These states correspond to the "time-reversal-invariant Majorana fermions" found in Ref. 13

58 C. R. Hu, Phys. Rev. Lett. 72, 1526 (1994).

59 The conclusions are the same mutatis mutandis for $g_{2}>0$.

${ }^{60}$ I. M. Lifshitz, Sov. Phys. JETP 11, 1130 (1960).

${ }^{61}$ G. E. Volovik, Quantum phase transitions from topology in momentum space, in: Quantum Analogues: From Phase Transitions to Black Holes and Cosmology, eds. W. G. Unruh and R. Schützhold, Springer Lecture Notes in Physics 718, 31 (2007).

62 G. E. Volovik, Exotic Properties of Superfluid ${ }^{3} \mathrm{He}$ (World Scientific, Singapore, 1992).

${ }^{63}$ G. E. Blonder, M. Tinkham, and T. M. Klapwijk, Phys. Rev. B 25, 4515 (1982).

${ }^{64}$ L. W. Molenkamp, G. Schmidt, and G. E. W. Bauer, Phys. Rev. B 64, 121202(R) (2001).

${ }^{65}$ U. Zülicke and C. Schroll, Phys. Rev. Lett. 88, 029701 (2001).

66 T. Matsuyama, C.-M. Hu, D. Grundler, G. Meier, and U. Merkt, Phys. Rev. B 65, 155322 (2002).

${ }^{67}$ K.-W. Lee and W. E. Pickett, Phys. Rev. B 72, 174505 (2005).

68 A. P. Schnyder, S. Ryu, and A. W. W. Ludwig, Phys. Rev. Lett. 102, 196804 (2009).

69 A. P. Schnyder, P. M. R. Brydon, D. Manske, and C. Timm, Phys. Rev. B 82, 184508 (2010).

${ }^{70}$ P. Hosur, S. Ryu, and A. Vishwanath, Phys. Rev. B 81, 045120 (2010).

71 Y. Asano and S. Yamano, Phys. Rev. B 84, 064526 (2011). 